\title{
Effect of using RT-44 as phase-change material in the porous microchannel
}

Mahdi Alibeigi ( $\nabla$ el.m.a.beigi@gmail.com )

Arak University of Technology https://orcid.org/0000-0003-0676-7141

Mohammadreza Sabzeali

Arak University of technology

Original Article

Keywords: Micro-channel, Porous media, Heat transfer, Nano-fluid, PCM, monte Carlo

Posted Date: February 5th, 2021

DOl: https://doi.org/10.21203/rs.3.rs-203976/v1

License: (9) This work is licensed under a Creative Commons Attribution 4.0 International License. Read Full License 


\title{
Effect of using RT-44 as phase-change material in the porous microchannel
}

\author{
Mahdi Alibeigi ${ }^{\mathrm{a},}{ }^{*}$, Mohamadreza Sabzehalib \\ a,b Department of Mechanical Engineering, Arak University of Technology, Arak 38181-46763, Iran \\ * Corresponding Author's Email: el.m.a.beigi@gmail.com;
}

\section{ABSTRACT}

In this paper, the influence of the utilizing RT-44 which, knows as a phase-change material (PCM) has used in an overall porous layer channel or optional additive nano particle of water/ZnO with three injecting fluid from its lower wall is investigated. The boundary condition slip-walls for the lower and higher walls of the micro-channel as insulation and constant temperature is considered. respectively, for assessment of PCM and nanoparticles consequences, the volume fraction of $0,2 \%$ and $5 \%$ determined for both of nanofluid and PCM. The result show that the Nusselt number increased with adding PCM to the porous media and decreasing with adding nanoparticles. When volume fraction of nano fluid is $5 \%$ the relative Nusselt number was calculated for PCM volume fraction of $1 \%, 2 \%$ and $5 \%$ with amount of $N u^{*}=0.9607, N{ }^{*}=0.9710$ and $N u^{*}=0.9869$ .respectively, for more supplementary the microchannel has levelized in two stage and three stage. The bulk temperature calculated for three stages for each level, at first stage was $299.90 \mathrm{~K}$, at second stage was $302.38 \mathrm{~K}$ and $302.72 \mathrm{~K}$ at third stage. For considering the geometric parameters effect an optimization with monte Carlo(MC) method has been simulated in terms of maximizing Nusselt number, it approved the relative Nusselt number Nusselt 1.026 and it increasing $0.26 \mathrm{~K}$ at the end of micro channel. Finally, the Additive PCM to porous material purposed for heating and additive nanofluid to the porous media purposed for cooling as well, the multistage channel purposed for heating at the latest stage.

Keywords: Micro-channel ,Porous media ,Heat transfer,Nano-fluid,PCM, monte Carlo 


\begin{tabular}{|c|c|c|c|}
\hline \multicolumn{2}{|c|}{ NOMENCLATURE } & \multirow[b]{2}{*}{$\theta$} & \multirow[b]{2}{*}{ dimensionless Temperature profile } \\
\hline$x$ & length distance $(\mathrm{m})$ & & \\
\hline$X$ & dimensionless length & $\varepsilon$ & porosity \\
\hline Y & dimensionless height & $\varphi$ & Volume fraction of nanoparticle \\
\hline$y$ & height distance $(\mathrm{m})$ & $\psi$ & Volume fraction of phase-change material \\
\hline$u$ & Horizontal components of velocity $(\mathrm{m} / \mathrm{s})$ & Subscripts & \\
\hline$v$ & Vertical components of velocity $(\mathrm{m} / \mathrm{s})$ & $a v g$ & average \\
\hline$H$ & hydraulic diameter of channel & eff & effective \\
\hline$T$ & Temperature $\left({ }^{\circ} \mathrm{C}\right)$ & $n f$ & nano-fluid \\
\hline$p$ & Pressure(Pa) & $s$ & slip \\
\hline$C_{p}$ & specific heat $(\mathrm{J} / \mathrm{kg} / \mathrm{K})$ & $S$ & solid \\
\hline$h$ & convection coefficient $\left(\mathrm{W} / \mathrm{m}^{2} / \mathrm{K}\right)$ & $p$ & porous \\
\hline$N u$ & Nusselt number & $b$ & Boltzmann \\
\hline $\operatorname{Pr}$ & prandtl number & $c$ & inlet boundary conditions \\
\hline $\operatorname{Re}$ & Reynolds number & $d$ & injection boundary conditions \\
\hline$D a$ & darcey number & $b f$ & Base fluid \\
\hline \multicolumn{2}{|c|}{ Greek Symbols } & Superscript & \\
\hline$\rho$ & Density $\left(\mathrm{kg} / \mathrm{m}^{3}\right)$ & $*$ & $\begin{array}{l}\text { dimensionless superscript geometrical } \\
\text { parameters }\end{array}$ \\
\hline$\mu$ & Viscosity(Pa.s) & & \\
\hline$\kappa$ & Permeability $\left(m^{2}\right)$ & & \\
\hline$\alpha$ & thermal diffusion $\left(\mathrm{m}^{2} / \mathrm{s}\right)$ & & \\
\hline$\beta$ & slip coefficient $(m)$ & & \\
\hline
\end{tabular}

\section{INTRODUCTION}


In recent decades, study of micro-channel intends many researchers cause of tremendous heat transfer effects and microfluidic attends by adding nano-fluid, porous-medium or a new material with the immense enhanced heat transfer, which is practical applying in devices as biological and chemical detections, drug delivery, and micro-mixing, also it can be used as a heat exchanger in the instruments such as heat sinks, steam methane reforming reactor reactors, nanoelectromechanical systems(NEMS)-based, microelectromechanical systems(MEMS) ,MHD areas and plate-pin heat exchanger.[1-4] scaling up microchannel process technology to commercial-scale is important which Focusing on and solving challenges around device fabrication, flow distribution and catalyst integration are the keys to success where Manufacturing steps demonstrated, including completion of a one eighth commercial-scale SMR reactor block. [5]demonstrated analysis NEMS-based and MEMS-based SMR devices provided extremely high sensitivity, sufficient to access new and interesting regimes for bio detection.[6]. Microchannel heat sinks integrated with heaters and temperature microsensors fabricated using a unique technique of mask-less and self-aligned silicon etching between bonded wafers where the heat sink performance under forced convection conditions ,where with CHF condition developed [7] .numerically investigated enhancing the heat transfer through a porous MCHS by enlarging the width or height of the channel where overall thermal resistance of a porous MCHS was not necessarily smaller than that without porous media[8] . an investigation reported the preparation of porous PDMS layers fabricated inside the microchannel of a microfluidic structure where, expected that the porous layers and blocks of PDMS can be used to immobilize different reagents, e.g. enzymes, or to prepare filtering membranes inside microfluidic structures. [9]The first investigation of the heat transfer of nanofluids were reported by Masuda et al[10]. Seetharamu et al. investigated the energy transport by applying the DarcyBrinkman flow in a local thermal non-equilibrium (LTNE) case with the thermal behavior of the porous- fluid system[11]. Finding the best nanoparticle from nanofluid in MCHS by least square and numerical methods based on saturated porous medium found[12]. A 3D space purposed microchannel at laminar flow with working fluid water nanofluid/GNP-SDBS (graphene nanoplatelet-sodium dodecylbenzene sulfonate) with solid nanoparticles mass fraction in range of $0-0.1 \%$ and for possibility of increasing average Nusselt number for each Reynolds number in range of 50-1000 via finite volume method was investigated.[13] 
the effects of solid phase heat generation on the entropy generation of water-Al2O3 nanofluid flow in asymmetrically heated porous microchannel developed an analytical model according to first law and second law of thermodynamics shows that the minimization of entropy generation. The intensification of solid-phase heat generation further reduces the discrepancy between the two models to less than $1 \%[14]$. represented nanofluid flow through a horizontal porous microchannel was scrutinized for effects of MHD field and heat generation in the solid on the generated entropy by the heat transfer processes with a wide variety of Re and Bejan number variations[15].

Accordingly, exploring of the previous literature with delicacy, many researches has been studied on the practical of the microchannel with different material on the heat transfer of the microchannels. In this study, the effect of diverting the phase-change material with porous medium with optional using of nanofluid microchannel. For more investigation, geometric optimization has done by Monte Carlo method for maximization the Nusselt number in concept bounded, also the microchannel levelized in one stage, two stage and three stage for discovering the best way of enhancing the heat transfer. All of the hypothesis channels have three jets and covered by porous-medium layer.

\section{Materials and Methods.}

In this case, several two-dimensional micro-channel by mixing aluminum porous foam as a porous media and phase-change material (PCM) and injective inlet velocity in three jets with velocity of $u_{d}=0.5 u_{c}$, where $u_{d}$ is the injective velocity and $u_{c}$ is the inlet velocity of microchannel.as well for the boundary temperature of these microchannel $T_{c}$ is the inlet temperature of ambient which defined as $293 \mathrm{~K}$ and $T_{h}$ is constant wall temperature which presumed for these microchannel as $303 \mathrm{~K}$. The influence parameters of geometry which can be changed .primarily, They have been assumed for the micro-porous channel for instance $\tau, H, L, D$ and $\lambda$ which are, the thickness of porous media, microchannel height, microchannel length, the diameter of each parallelogram jets and $\lambda$ the angular injection respectively, the diameter defined as $D=0.001 L$ and $\lambda=45$ in these microchannel the thickness of porous media equals to the height of total channel for covering the all over the channel by aluminum foam. The PCM and nanofluid add to the porous layer full covered for the comparison of heat transfer enhancement. Then levelized microchannel investigated in three case a) one stage b) two stage c) three stage which shown in fig 1.

a) b) c) 

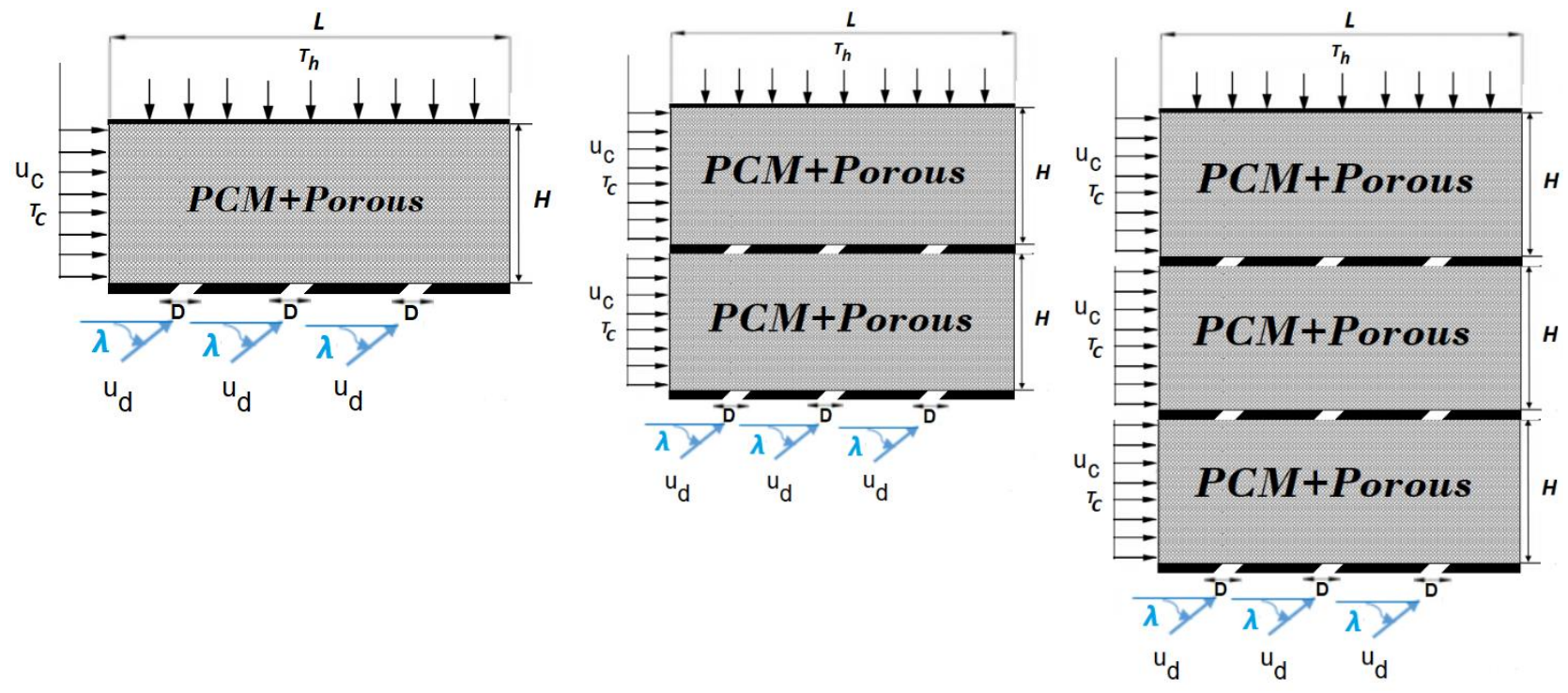

Figure 1. Schematic of microchannel a)one stage b) two stages c)three stages.

The basically governing equation such as Momentum, Continuity and energy equations can be reviewed as,

$$
\begin{aligned}
& u \frac{\partial u}{\partial x}+v \frac{\partial u}{\partial y}=-\frac{\partial p}{\partial x}+\frac{1}{\operatorname{Re}}\left(\frac{\partial^{2} u}{\partial x^{2}}+\frac{\partial^{2} v}{\partial y^{2}}\right) \\
& \frac{\partial u}{\partial x}+\frac{\partial v}{\partial y}=0 \\
& u \frac{\partial T}{\partial x}+v \frac{\partial T}{\partial x}=-\frac{\partial p}{\partial x}+\frac{1}{\operatorname{Re} \operatorname{Pr}}\left(\frac{\partial^{2} T}{\partial x^{2}}+\frac{\partial^{2} T}{\partial y^{2}}\right)
\end{aligned}
$$

The dimensionless parameters habitually utilized such as dimensionless geometric parameters, dimensionless slip coefficient, dimensionless velocities, dimensionless drop pressure, temperature profile, Reynolds number, Prandtl number, Nusselt number and Darcey number. Orderly defined as,

$$
\begin{aligned}
& X=\frac{x}{H}, L^{*}=\frac{L}{H}, \beta^{*}=\frac{\beta}{H} \\
& Y=\frac{y}{H}, H^{*}=\frac{H_{0}}{H}, \tau^{*}=\frac{\tau}{H}
\end{aligned}
$$




$$
\begin{aligned}
& U=\frac{u}{u_{c}}, V=\frac{v}{v_{c}}, U_{d}=\frac{u_{d}}{u_{c}}, V_{d}=\frac{v_{d}}{v_{c}}, P=\frac{\Delta p}{\rho_{n f} u_{c}^{2}} \\
& \theta=\frac{T-T_{c}}{T_{h}-T_{c}} \cdot \operatorname{Re}=\frac{u_{c} H}{v_{f}} \operatorname{Pr}=\frac{v_{f}}{\alpha_{f}} \\
& N u(x)=\frac{h D_{h}}{k} \\
& D a=\frac{\kappa}{D_{h}^{2}}
\end{aligned}
$$

Where $D_{h}, \mathrm{~h}, k$ and $\kappa$ are the hydraulic diameter, heat transfer convection coefficient, thermal conductivity.

The boundary condition for each stage determined in following equations.

$$
U_{c}=1, \quad V=0, \quad \theta=0 \quad \text { For } \quad X=0,0 \leq Y \leq 1
$$

$$
\frac{\partial U}{\partial X}=0, \quad V=0, \quad \frac{\partial \theta}{\partial Y}=0 \quad \text { For } \quad X=L^{*}, 0 \leq Y \leq 1
$$

$$
\begin{aligned}
& U_{S}=\beta^{*}\left(\frac{\partial U}{\partial Y}\right)_{Y=0}, \quad V=0, \quad \frac{\partial \theta}{\partial Y}=0 \quad \text { For } \quad Y=0,0 \leq X \leq L^{*} \\
& U_{S}=\beta^{*}\left(\frac{\partial U}{\partial Y}\right)_{Y=1}, \quad V=0, \quad \theta=1 \quad \text { For } \quad Y=1,0 \leq X \leq L^{*}
\end{aligned}
$$

Presentively, adding porous material to the micro-channel has discussed the dimensionless momentum equation when the porous medium has used in the microchannel should be calculated by Brinkman ,Darcey frochimeters momentum equation such as, [20]

$$
-\frac{\partial P}{\partial X}+\mu_{e f f} \frac{\partial^{2} U}{\partial Y^{2}}+\frac{\mu_{f}}{\kappa} U=0
$$

The conservation energy in the porous medium is:

$$
\nabla \cdot\left(\left(\rho c_{p}\right)_{e f f} u T\right)=\nabla \cdot\left(k_{e f f} \nabla T\right)
$$

Where, $\mu_{\text {eff }}$ is the effective dynamic viscosity of the porous medium. 
The effective Nusselt can be determined with as a function of gradient temperature profile and a factor of $\frac{k_{e f f}}{k_{b f}}$ in uppers walls.

$$
N u=-\left.\frac{k_{e f f}}{k_{b f}} \frac{\partial \theta}{\partial Y}\right|_{Y=H^{*}}
$$

formerly, the slip velocity $U_{S}$ has been calculated with the Brownian motion[21].

$$
U_{s}=\frac{2 k_{b} T}{\pi \mu_{b f} d_{S^{2}}}
$$

Where the Boltzmann constant $k_{b}=1.3807 \times 10-23 \mathrm{~J} / \mathrm{K}$ and $d_{S}=40 \mathrm{~nm}$, is solid nanoparticles diameter.

In each stage, the boundary condition should be defined same as first stage where can be find in the following formula.

the boundary conditions of the injection jets bounded by,

$$
U_{d}=0.5 \cos (\alpha), \quad V_{d}=0.5 \cos (\alpha), \quad \theta=0
$$

According to the mixture modeling, the effective thermophysical properties can be obtained by using equations in table 1.

The thermophysical properties of nanofluid such as $\rho_{n f}, c_{p, n f}, K_{n f}, \mu_{n f}$ gotten in table1, also, the thermophysical properties of porous material which has been used can be find in table2, lastly,

\begin{tabular}{|c|c|c|c|c|}
\hline Volume fraction & $\phi=0$ & $\phi=1 \%$ & $\phi=3 \%$ & $\phi=5 \%$ \\
\hline$\rho_{\text {Density }}\left(k g / m^{3}\right)$ & 998.2 & 1043.1 & 1135.2 & 1037.6 \\
\hline$\mu_{n f}(p a . s)$ & $8.9 E-4$ & $9.1265 E-4$ & $9.6042 E-4$ & 0.0010118 \\
\hline$c_{p \text { specific Heat }}, n f(J / k g . K)$ & 4182 & 3981.2 & 3633.8 & 3338.5 \\
\hline
\end{tabular}
the thermophysical properties of PCM materials has been given in table 2 .

Table 1. Thermophysical properties of nano-fluid Zno/Water.[22] 
$k_{n f}(W / m . k)$

Table 2. Thermophysical properties of porous material and RT-44 as PCM material.[3, 23]

\begin{tabular}{|c|c|c|c|}
\hline \multicolumn{2}{|c|}{ Porous material thermophysics properties } & \multicolumn{2}{|c|}{ PCM thermophysics properties } \\
\hline$\rho_{p}\left(k g / m^{3}\right)$ & 400 & $\underbrace{\rho_{P C M}\left(\mathrm{~kg} / \mathrm{m}^{3}\right)}_{\text {Density }}$ & 771 \\
\hline $\begin{array}{c}c_{p}, p(J / k g . K) \\
\text { Specific Heat }\end{array}$ & 400 & $\begin{array}{c}c_{p}{ }^{\prime}{ }_{P C M}(J / k g . K) \\
\text { Specific Heat }\end{array}$ & 2000 \\
\hline $\begin{array}{l}{ }_{p}^{k}(W / m \cdot k) \\
\text { Conductivity }\end{array}$ & 5.8 & $\begin{array}{c}k_{P C M}(W / m . k) \\
\text { Conductivity }\end{array}$ & 0.2 \\
\hline $\begin{array}{l}\kappa\left(m^{2}\right) \\
\text { Permeability }\end{array}$ & $10^{-5}$ & - & - \\
\hline $\begin{array}{c}\varepsilon_{p} \\
\text { Porosity }\end{array}$ & 0.8 & - & - \\
\hline
\end{tabular}

The effective thermal properites. When, nano fluid, Porous material and pcm use in the microchannel display in table 3. For each supplimenatry.

Table 3. effectivenessThermophysical properties for each supplimentary.

\begin{tabular}{|c|c|c|c|c|c|}
\hline \multicolumn{2}{|l|}{$k_{\text {eff }}$} & \multicolumn{2}{|l|}{$\rho_{\text {eff }}$} & \multicolumn{2}{|l|}{$C_{\text {Peff }}$} \\
\hline$k_{e f f, n f}=(1-\varphi) k_{b f}+\varphi k_{s}$ & (19) & $\rho_{e f f, n f}=(1-\varphi) \rho_{b f}+\varphi \rho_{s}$ & (22) & $\left(\rho c_{p}\right)_{e f f, n f}=(1-\varphi)\left(\rho c_{p}\right)_{b f}+\varphi\left(\rho c_{p}\right)_{s}$ & (25) \\
\hline$k_{e f f, p m}=\left(1-\varepsilon_{p}\right) k_{p m}+\varepsilon_{p} k_{n p}$ & (20) & $\rho_{e f f, p m}=\left(1-\varepsilon_{p}\right) \rho_{p m}+\varepsilon_{p} \rho_{n p}$ & (23) & $\left(\rho c_{p}\right)_{e f f, n f}=(1-\varphi)\left(\rho c_{p}\right)_{b f}+\varphi\left(\rho c_{p}\right)_{s}$ & (26) \\
\hline$k_{e f f, P C M}=(1-\psi) k_{b f}+\psi k_{P C M}$ & (21) & $\rho_{e f f, P C M}=(1-\psi) \rho_{b f}+\psi \rho_{P C M}$ & (24) & $\left(\rho c_{p}\right)_{e f f, n f}=(1-\psi)\left(\rho c_{p}\right)_{b f}+\psi\left(\rho c_{p}\right)_{s}$ & (27) \\
\hline
\end{tabular}

Where, $\varphi$ is the nano-fluid volume fraction, $\psi$ is the PCM volume fraction and $\varepsilon_{p}$ Is the porosity, Nano particle has been chosen $\mathrm{ZnO}$ and has mixed with water for assembling of nano-fluid of $\mathrm{ZnO} /$ Water the thermophysical properties, such as, $\rho_{S}, c_{p, s}, k_{s}$ orderly are $5600 \mathrm{~kg} / \mathrm{m}^{3}, 495 \mathrm{~J} / \mathrm{kg} . \mathrm{K}, 13.00 \mathrm{~W} / \mathrm{m} . \mathrm{k}$.

$\mu_{n f}$ is Another thermophysical property. whereas the dynamic viscosity of nano-fluid which, it can be determined with different $\varphi$ as a function of nano-fluid volume fraction and dynamic viscosity of base fluid which, previously identified as Brownian equation which can be discovered in following equation[21, 22],

$$
\mu_{n f}=\frac{\mu_{b f}}{(1-\varphi)^{2.5}}
$$


$\alpha_{e f f}$ is the thermal diffusion, which can be calcualted as follow.

$$
\alpha_{e f f}=\frac{k_{e f f}}{\left(\rho C_{p}\right)_{e f f}}
$$

Average value of nusselt and slip velocity can be calculated with integerial application in following equation ,

$$
\begin{aligned}
& N u_{a v g}=\frac{1}{L} \int_{0}^{L} N u(x) d x \\
& u_{s_{a v g}}=\frac{1}{L} \int_{0}^{L} u_{s} d x
\end{aligned}
$$

relative value of local nusselt number and relative slip velocity can be presented with comparision of a base local nusselt number and base amount of slip velocity application in following equation ,

$$
\begin{gathered}
N u^{*}=\frac{N u}{N u_{0}} \\
u_{s}^{*}=\frac{u_{s}}{u_{s_{0}}}
\end{gathered}
$$

Finite Element method (FEM) is a influential numerical technique where multifaceted geometries and material heterogeneities.outstanding of numerous restrictions in FEM technique such as large computational competences habitually is desirable for such actions, the usage of FEM within optimized models is so far a inspiring command.

The technique coding of COMSOL commericial software is a FEM scheme as numerical analysis with various physics and applicant engineering modules which could be coupled as with an integrated user interface,as weel as multiphysics arrangement.which permits users to contribution the different modules, which coupled the partial differential equations

(PDE) directly. Besides, the COMSOL code application producer can be utilized to concept dedicated applications founded on the physics model. The adaptive mesh refinement choice could be applied the intensification of correctness of the solution by increasing the number of elements in the areas of largest numerical error, thus reducing the spatial discretization errors of the results.

implementation of COMSOL models within Monte Carlo (MC) optimization procedures.

MC optimazed tecnique operated as a random number generator to generate the input distributions where used in this sutdy.[24-26]

For more empathetic of methodology the flowchart delivered in fig 4. 


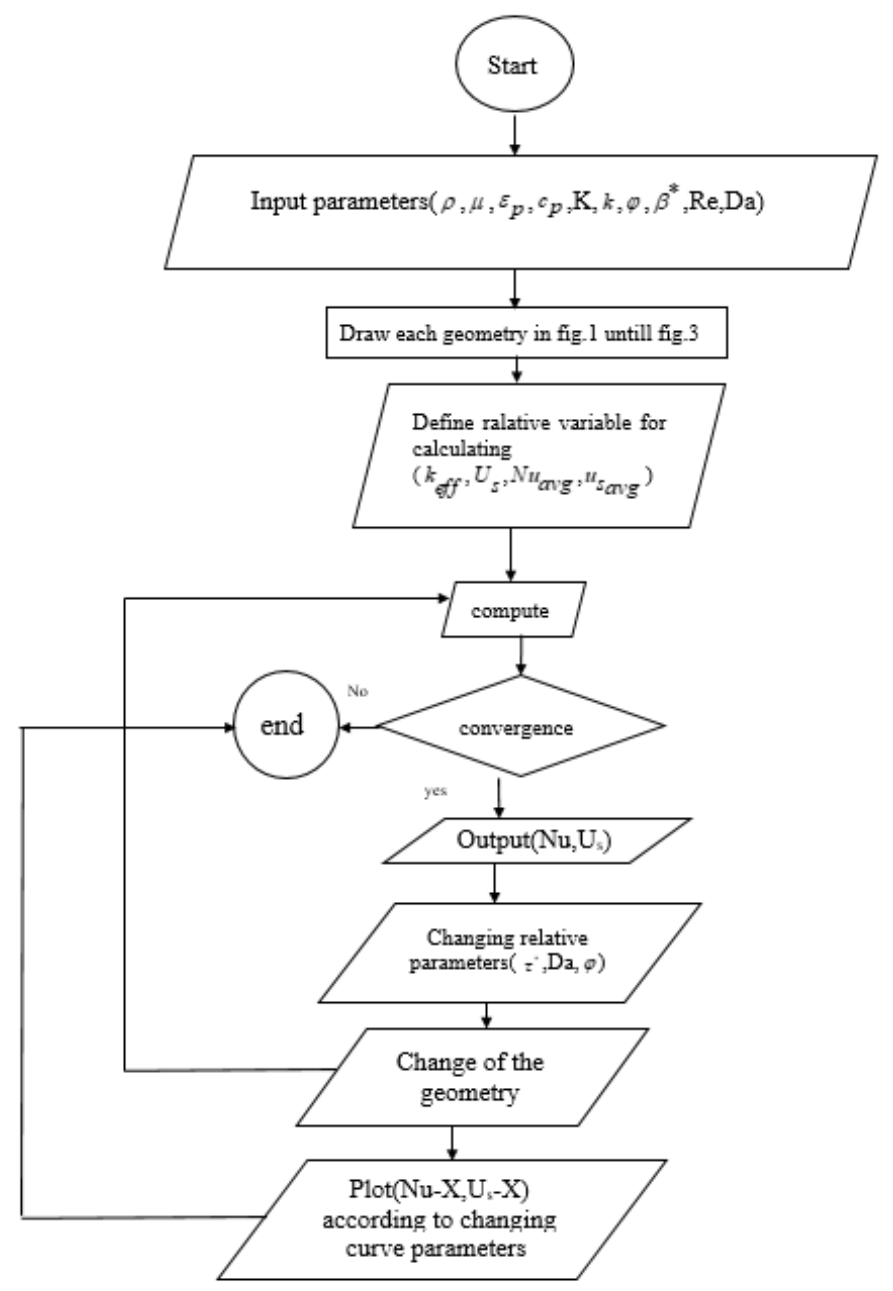

Figure 2. flowchart of methodology.

\section{Results}

In this case, the usage effect of PCM in porous medium a two-dimensional micro-channel on dimensionless heat transfer number which named Nusselt number is investigated. To verify of this numeric correlation has validated with the same literature, which has done in 2020 by Alibeigi and Farahani [3] this analogy reproved of the numerical analyses in this scope, the consequences for the case where the Reynolds number is 100 and illustrated in Fig.5. At this point is a moral match between the results of the present study and the reference and there is less than $10 \%$ difference. 


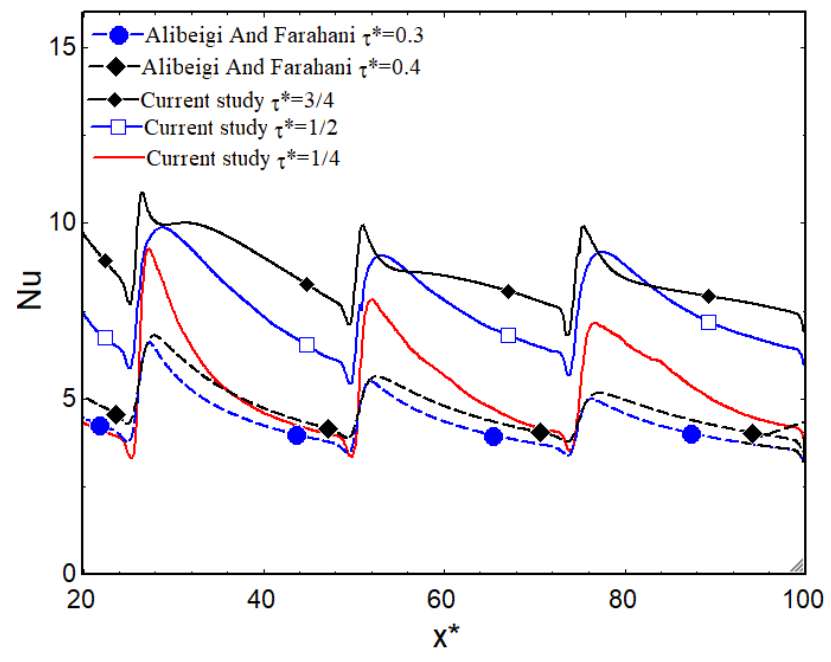

Figure 3. Validation of current study with similar study.

The discrepancy of slip coefficient in slip velocity and local Nusselt number with the changing Reynolds number in Figures 4-9 displays for the covering the total microchannel without additive PCM with porous medium, velocity and Nusselt number changes with variation of Reynolds number consist of 10,100 and 500 and slip coefficient $\beta=0.01, \beta=0.05$ and $\beta=0.1$ at $\tau^{*}=1$ of the porous layer located to the microchannel with single stage the relative slip velocity rather than the base velocity in condition of $\beta=0.01$ and $\operatorname{Re}=10$ were calculated.at the $\operatorname{Re}=10$ for $\beta=0.05$ and $\beta=0.1$ the relative slip velocity orderly calculated in average of $u_{s}{ }^{*}=0.1695$ for $\beta=0.05$ and $u_{s}{ }^{*}=0.6378$ for $\beta=0.1$.at $\operatorname{Re}=100$ for $\beta=0.05$ and $\beta=0.1$ the relative slip velocity orderly calculated in average of $u_{s}{ }^{*}=0.1631$ for $\beta=0.05$ and $u_{s}{ }^{*}=0.6349$ for $\beta=0.1$ at the $\operatorname{Re}=500$ for $\beta=0.05$ and $\beta=0.1$ the relative slip velocity orderly calculated in average of $u_{s}{ }^{*}=0.1626$ for $\beta=0.05$ and $u_{s}{ }^{*}=0.6347$ for $\beta=0.1$.it can be settled the effects of slip coefficient in the PCM microchannel with overall cover porous medium on the slip velocity was decreased by increasing the slip coefficient. Also, it can be calculated repeatedly for the local Nusselt in order to gain the average of relative Nusselt number in Porous microchannel without adding PCM. at the $\operatorname{Re}=10$ for $\beta=0.05$ and $\beta=0.1$ the relative Nusselt number orderly calculated in average of $\mathrm{Nu}^{*}=1.01$ for $\beta=0.05$ and $N u^{*}=1.03$ for $\beta=0.1$. at $\operatorname{Re}=100$ for $\beta=0.05$ and $\beta=0.1$ the relative slip velocity orderly calculated in average of $u_{s}{ }^{*}=0.1631$ for $\beta=0.05$ and $u_{s}{ }^{*}=0.6349$ for $\beta=0.1$ at the $\operatorname{Re}=500$ for $\beta=0.05$ and $\beta=0.1$ the relative slip velocity orderly calculated in average of $u_{s}{ }^{*}=0.1626$ for $\beta=0.05$ and $u_{s}{ }^{*}=0.6347$ for Volume fractions of $1 \%, 3 \%$, and $5 \%$ percent are taken. It can be realized that with increasing volume fraction, Nusselt number has been reduced $2.017 \%$ cause of the increasing $\mathrm{k}$ in the equation (24). The slip velocity has any changing with alternative of volume fraction. Volume fraction changes do not have a substantial effect on slip velocity. 


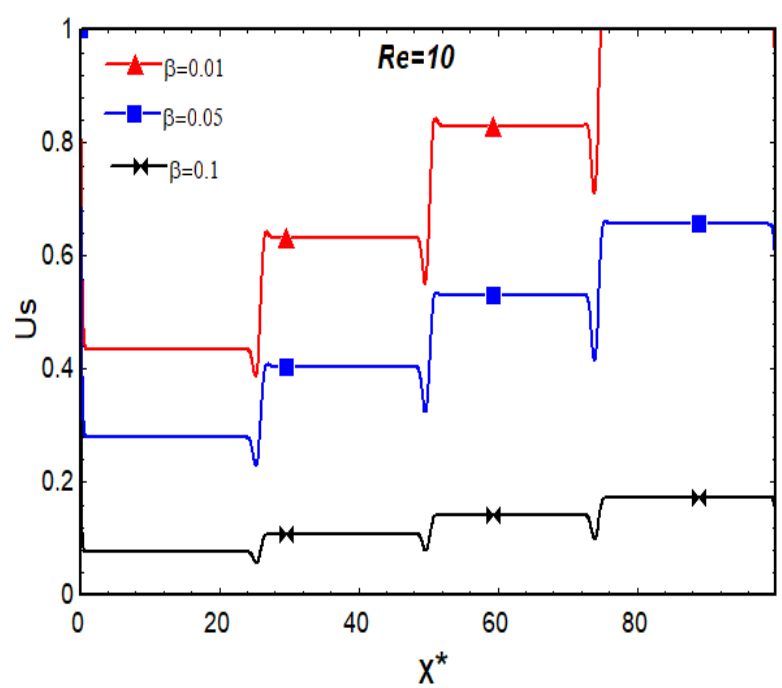

Figure 4. variations of slip velocity for $\mathrm{Re}=10$ in slip upper slip wall.

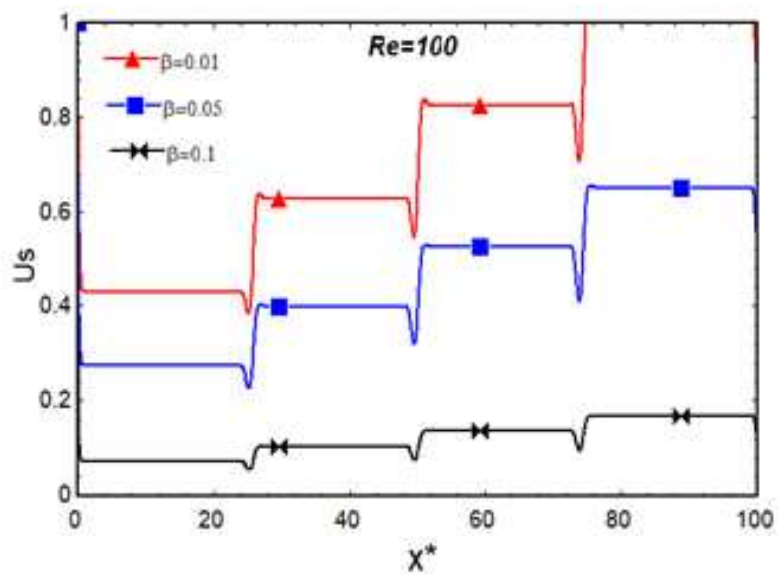

Figure 5. variations of slip velocity for $\mathrm{Re}=100$ in slip upper slip wall.

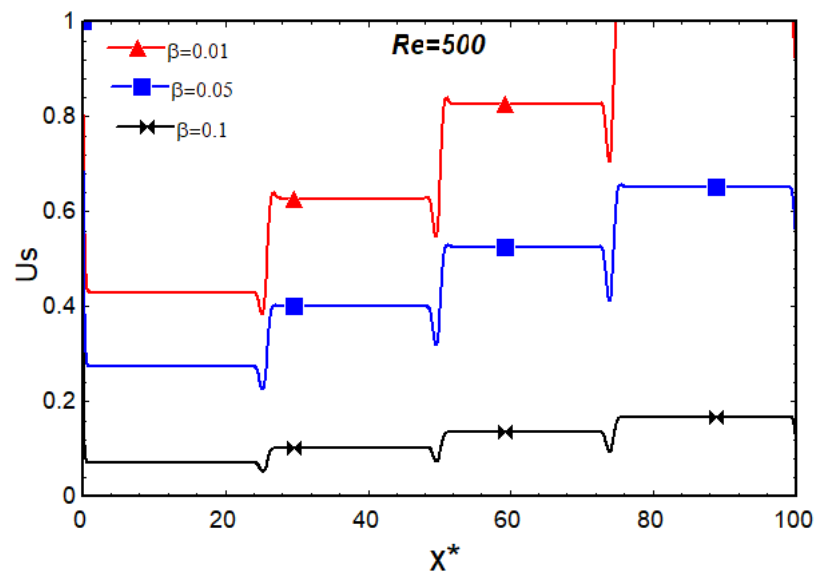


Figure 6. variations of slip velocity for $\mathrm{Re}=500$ in slip upper slip wall.

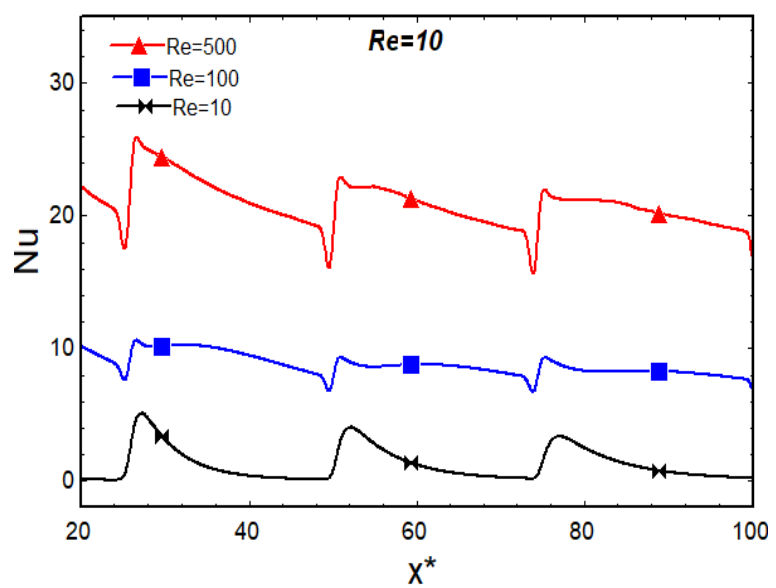

Figure 7. variations of Nusselt number for $\mathrm{Re}=10$ in constant temperature wall.

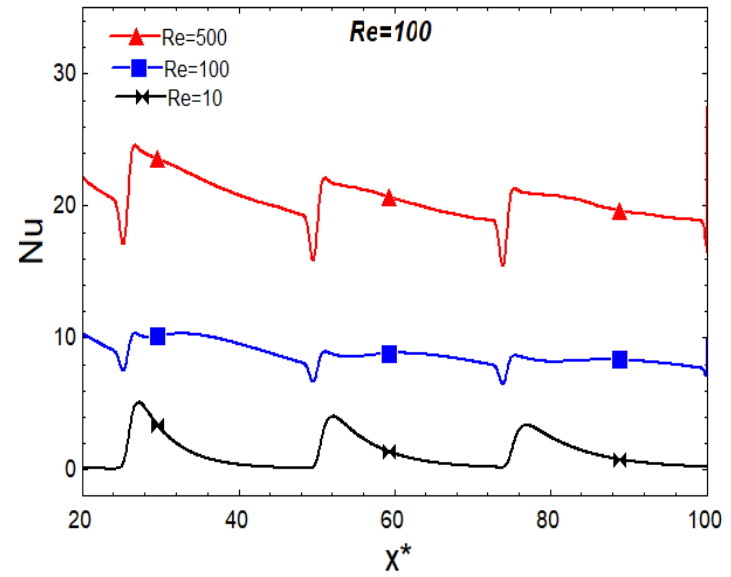

Figure 8. variations of Nusselt number for $\mathrm{Re}=100$ in constant temperature wall.

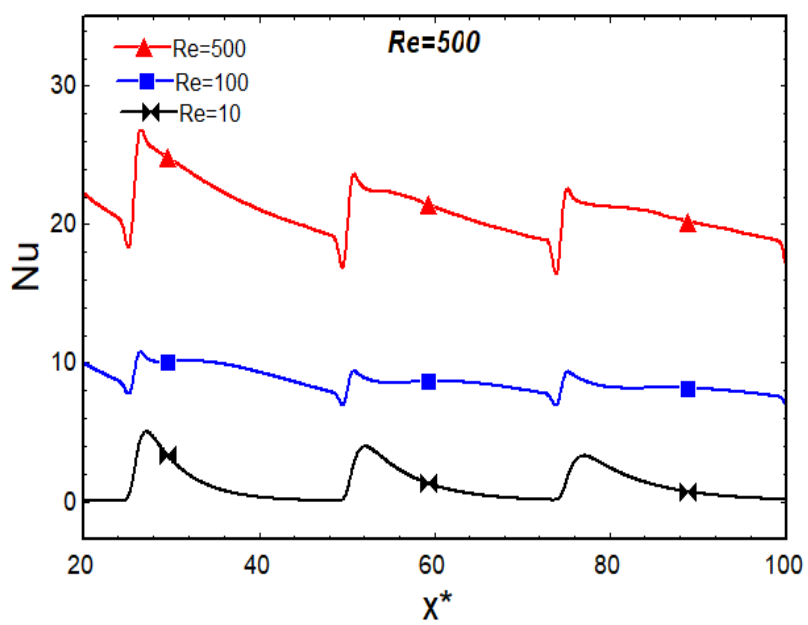

Figure 9. variations of Nusselt number for $\mathrm{Re}=500$ in constant temperature wall. 
In overall cover porous layer into the microchannel by adding the PCM and nanofluid the changes of local Nusselt number with the variation of PCM volume fraction and nonfluid volume fraction in amount of 0 as not existing PCM option or nanofluid option as well adding PCM with volume fraction of 2 and 5 or nanofluid with volume fraction of 2 and 5 to the overall covered porous layer microchannel. The results indicated in the figures 10-12.the base local Nusselt number considered 0 both volume fraction of PCM and nanofluid option. The deliberation of relative Nusselt number was calculated.at constant amount of volume fraction of nano fluid as $\varphi=0$ for variation of PCM volume fraction $\psi=2 \%$ and $\psi=5 \%$ the relative Nusselt number orderly was calculated $\mathrm{Nu}^{*}=1.009$ and $\mathrm{Nu}^{*}=1.023$. at constant amount of volume fraction of nano fluid as $\varphi=2 \%$ for variation of PCM volume fraction $\psi=0, \psi=2 \%$ and $\psi=5 \%$ the relative Nusselt number orderly was calculated $\mathrm{Nu}^{*}=0.9738, \mathrm{Nu}^{*}=0.9841$ and $\mathrm{Nu}^{*}=1.000$.

at constant amount of volume fraction of nano fluid as $\varphi=5 \%$ for variation of PCM volume fraction $\psi=0, \psi=2 \%$ and $\psi=5 \%$ the relative Nusselt number orderly was calculated $N u^{*}=0.9607, \mathrm{Nu}^{*}=0.9710$ and $\mathrm{Nu}^{*}=0.9869$. the effects of adding PCM and nanofluid showed PCM can be decreased the Nusselt number by increasing the volume fraction of nanofluid in order to use with total covered porous layer and nanofluid .also by increasing the volume fraction of PCM, the Nusselt number was increased.

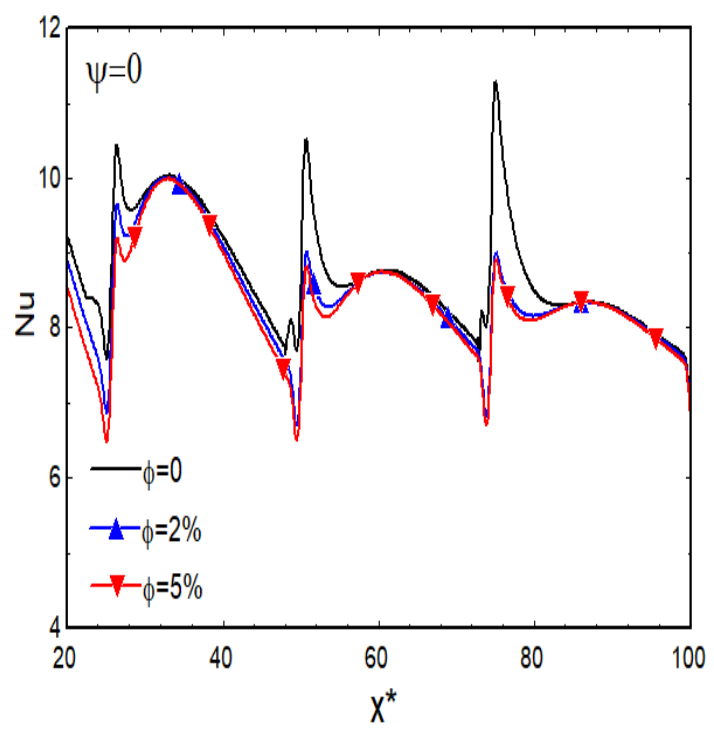

Figure 10. effects on nano particle volume fraction with $\psi=0$ on Nusselt number. 


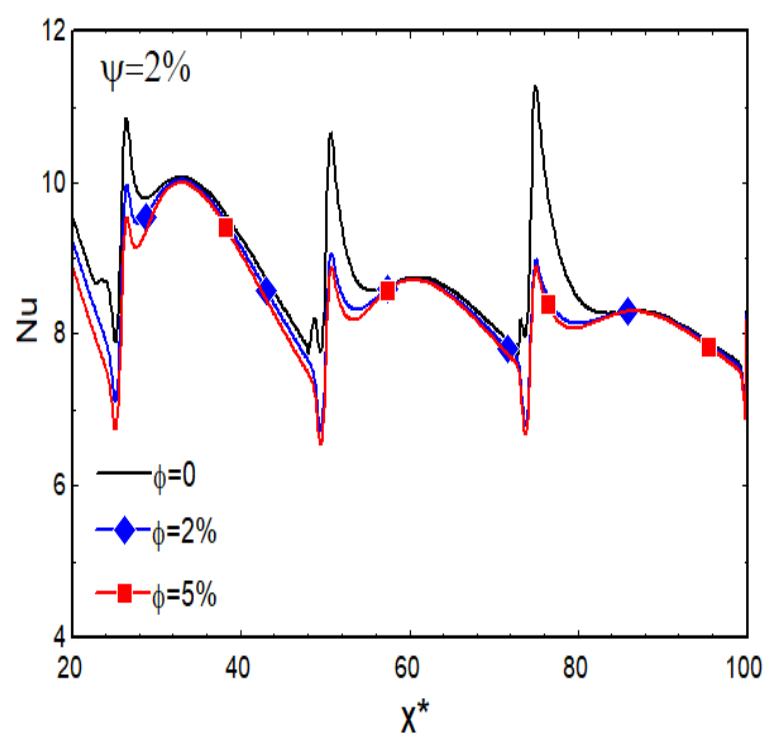

Figure 11. effects on nano particle volume fraction with $\psi=2 \%$ on Nusselt number.

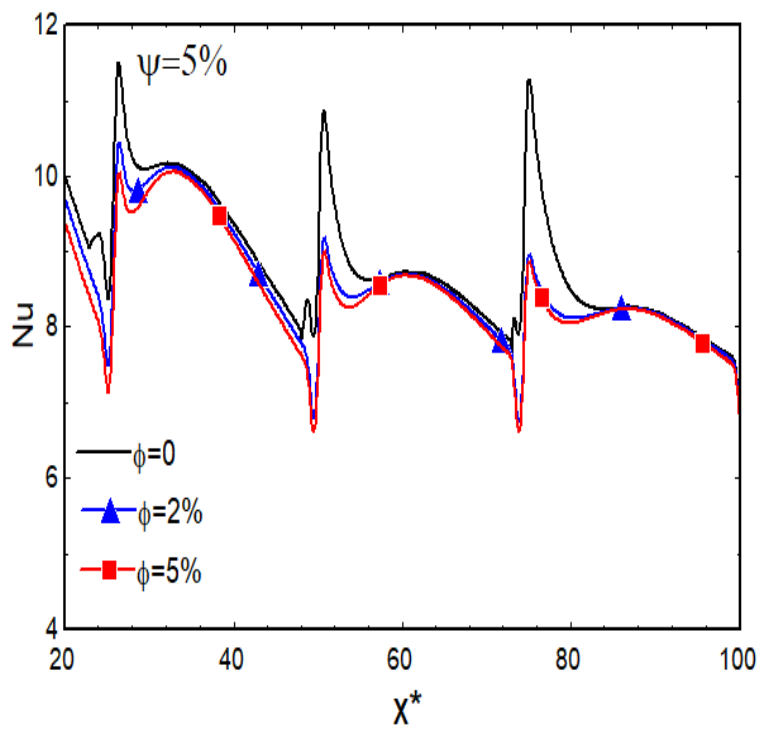

Figure 12. effects on nano particle volume fraction with $\psi=5 \%$ on Nusselt number.

Another novel microchannel with total porous layer covered adding the new stage to the top of micro channel with relevancy of jet injection. The three microchannel with One-stage, Two-stage and three-stage considered. The temperature counter of each stage showed in fig. at three stage, the bulk temperatures were calculated as $300.14 \mathrm{~K}$ in first stage, $302.46 \mathrm{~K}$ in the second stage and $302.72 \mathrm{~K}$ at third stage.at two stage, the bulk temperature was $299.87 \mathrm{~K}$ in first stage and $302.38 \mathrm{~K}$ in second stage. In first stage was $299.90 \mathrm{~K}$.it was approved by adding stage the bulk temperature of each stage has been increased. Also, the temperature counters indicated in fig 13. 


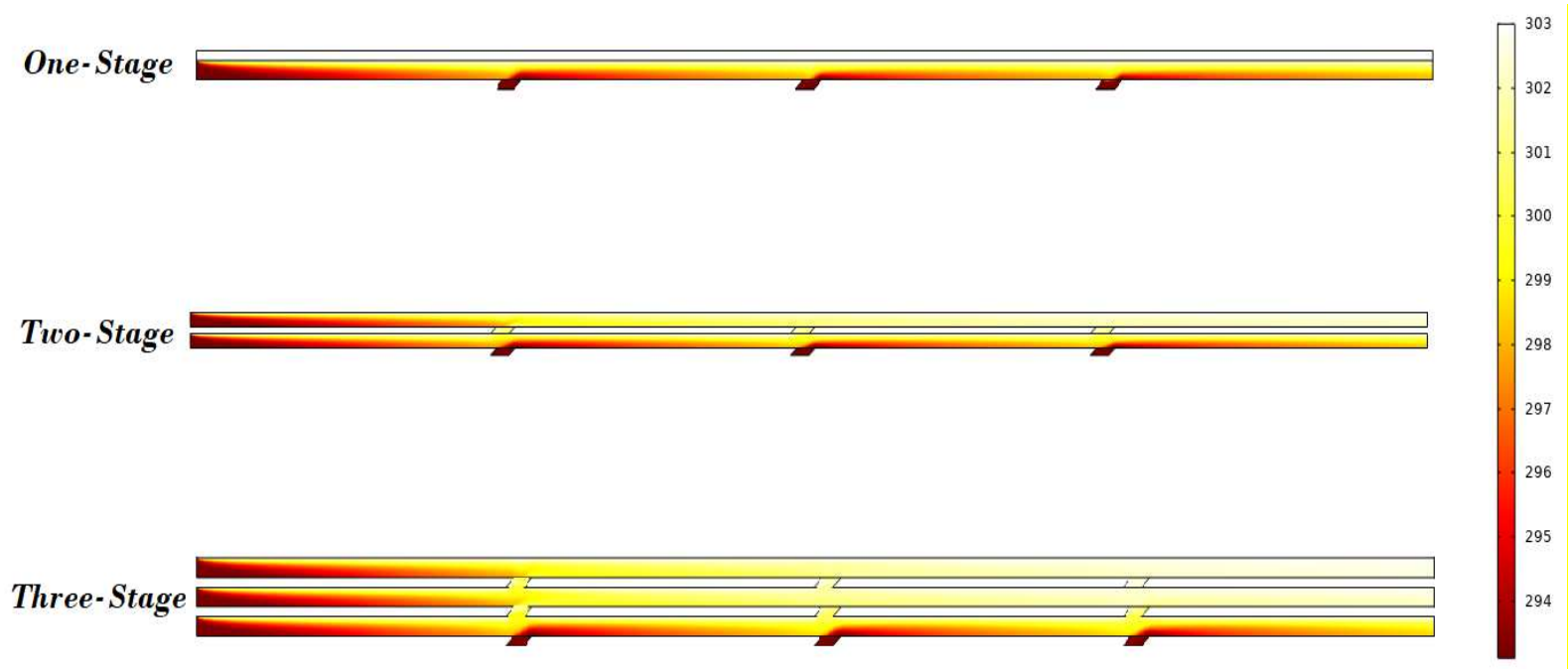

Figure 13. temperature counter for each stage.

The geometric parameters have effects of the performance of microchannel. Meant for this technique, optimization can be chosen for the discovery of the best performance in a selective bounded, as the earlier has discussed, optimization can be resolved the geometric parameters chosen by Mont Carlo method. The parameters chosen with their bound shown in table 4 . at induvial thermal properties and same conditions such constant density, constant thermal conductivity coefficient, constant Reynolds number, inlet Velocity and so on excepted the geometric variables. the geometric optimized microchannel enhanced with maximization Nusselt number, the bulk temperature in optimized channel calculated in terms of maximization of Nusselt number $300.06 \mathrm{~K}$ and relative Nusselt 1.026, it approved it has increased $0.26 \mathrm{~K}$ at the end of channel.

Table 4. parameters bounded for optimization.

\begin{tabular}{|l|l|l|}
\hline Parameters & $\begin{array}{l}\text { Lower } \\
\text { bounded }\end{array}$ & $\begin{array}{l}\text { Upper } \\
\text { bounded }\end{array}$ \\
\hline $\mathrm{H}$ & $0.5 \mathrm{HO}$ & $1.2 \mathrm{HO}$ \\
\hline$\beta$ & 0.05 & 0.1 \\
\hline \multicolumn{1}{|c|}{$L_{n}$} & $0.1 \mathrm{~L}$ & $0.2425 \mathrm{~L}$ \\
\hline $\mathrm{D}$ & $0.005 \mathrm{~L}$ & $0.02 \mathrm{~L}$ \\
\hline
\end{tabular}

Where, $\mathrm{HO}$ is the primary amount of microchannel height and $L_{n}$ is the distance between two jets. 


\section{4 .Conclusions}

In this study, the usefulness of using PCM and nanofluid of $\mathrm{ZnO}$ in the porous material within multi stage microchannels on heat transfer appearances was studied. A number of several volume fraction for both of PCM and $\mathrm{ZnO}$ at the porous layer were tested. The functional software was the Comsol software base on Darcy equation for porous media and Monte carlo method in order to optimize the geometry of microchannel with finite element method. The demonstration consequences with increasing the amount of $\varphi$, relative Nusselt number decreased. Also, effects of growing the amount of $\psi$ on relative Nusselt number was increasing the relative Nusselt number. correspondingly, it can be purposed by adding PCM to the porous media. It can be more suitable for heating and by adding nano fluid the channel should be more suitable for cooling. finally, the multistage channel purposed for heating at the latest stage. 


\section{REFERENCES}

1. Cao B-Y, Sun J, Chen M, Guo Z-Y. Molecular momentum transport at fluid-solid interfaces in MEMS/NEMS: a review. International journal of molecular sciences. 2009;10(11):4638-706.

2. Stone HA, Stroock AD, Ajdari A. Engineering flows in small devices: microfluidics toward a lab-on-a-chip. Annu Rev Fluid Mech. 2004;36:381411.

3. Alibeigi M, Farahani SD. Effect of Porous Medium Positioning on Heat Transfer of Micro-channel with Jet. International Journal of Engineering. 2020;33(10):2057-64. doi:10.5829/ije.2020.33.10a.24.

4. Jing $D$, Zhan X. Cross-sectional dimension dependence of electroosmotic flow in fractal treelike rectangular microchannel network. Micromachines. 2020;11(3):266.

5. Tonkovich A, Kuhlmann D, Rogers A, McDaniel J, Fitzgerald S, Arora R et al. Microchannel technology scale-up to commercial capacity. Chemical Engineering Research and Design. 2005;83(6):634-9.

6. Arlett J, Roukes M. Ultimate and practical limits of fluid-based mass detection with suspended microchannel resonators. Journal of Applied Physics. 2010;108(8):084701.

7. Jiang L, Wong $M$, Zohar Y. Phase change in microchannel heat sinks with integrated temperature sensors. Journal of microelectromechanical systems. 1999;8(4):358-65.

8. Hung $T-C$, Huang $Y-X$, Yan $W-M$. Thermal performance analysis of porous-microchannel heat sinks with different configuration designs. International Journal of Heat and Mass Transfer. 2013;66:235-43.

9. Juchniewicz M, Stadnik D, Biesiada K, Olszyna A, Chudy M, Brzózka Z et al. Porous crosslinked PDMS-microchannels coatings. Sensors and Actuators B: Chemical. 2007;126(1):68-72.

10. Masuda H, Ebata A, Teramae K, Hishinuma N, Ebata Y. Alteration of thermal conductivity and viscosity of liquid by dispersing ultra-fine particles (dispersion of $\mathrm{Y}$-Al2O3, SiO2 and TiO2 ultra-fine particles). 1993.

11. Seetharamu K, Leela V, Kotloni N. Numerical investigation of heat transfer in a micro-porous-channel under variable wall heat flux and variable wall temperature boundary conditions using local thermal non-equilibrium model with internal heat generation. International Journal of Heat and Mass Transfer. 2017;112:201-15.

12. Pourmehran O, Rahimi-Gorji M, Hatami M, Sahebi S, Domairry G. Numerical optimization of microchannel heat sink (MCHS) performance cooled by KKL based nanofluids in saturated porous medium. Journal of the Taiwan Institute of Chemical Engineers. 2015;55:49-68.

13. Khodabandeh E, Rozati SA, Joshaghani M, Akbari OA, Akbari S, Toghraie D. Thermal performance improvement in water nanofluid/GNP-SDBS in novel design of double-layer microchannel heat sink with sinusoidal cavities and rectangular ribs. Journal of Thermal Analysis and Calorimetry. 2019;136(3):1333-45. doi:10.1007/s10973-018-7826-2.

14. Ting TW, Hung YM, Guo N. Entropy generation of viscous dissipative nanofluid convection in asymmetrically heated porous microchannels with solid-phase heat generation. Energy Conversion and Management. 2015;105:731-45.

15. Hosseini S, Ghasemian M, Sheikholeslami M, Shafee A, Li Z. Entropy analysis of nanofluid convection in a heated porous microchannel under MHD field considering solid heat generation. Powder technology. 2019;344:914-25.

16. Arabpour A, Karimipour A, Toghraie D, Akbari OA. Investigation into the effects of slip boundary condition on nanofluid flow in a double-layer microchannel. Journal of Thermal Analysis and Calorimetry. 2018;131(3):2975-91. doi:10.1007/s10973-017-6813-3.

17. Sarafraz MM, Tlili I, Tian Z, Khan AR, Safaei MR. Thermal analysis and thermo-hydraulic characteristics of zirconia-water nanofluid under a convective boiling regime. Journal of Thermal Analysis and Calorimetry. 2020;139(4):2413-22. doi:10.1007/s10973-019-08435-x.

18. Mashayekhi R, Khodabandeh E, Akbari OA, Toghraie D, Bahiraei M, Gholami M. CFD analysis of thermal and hydrodynamic characteristics of hybrid nanofluid in a new designed sinusoidal double-layered microchannel heat sink. Journal of Thermal Analysis and Calorimetry. 2018;134(3):2305-15. doi:10.1007/s10973-018-7671-3.

19. Heydari A, Akbari OA, Safaei MR, Derakhshani M, Alrashed AAAA, Mashayekhi R et al. The effect of attack angle of triangular ribs on heat transfer of nanofluids in a microchannel. Journal of Thermal Analysis and Calorimetry. 2018;131(3):2893-912. doi:10.1007/s10973-017-6746-x.

20. Nield DA, Bejan A. Convection in porous media. Springer; 2006.

21. Brinkman H. The viscosity of concentrated suspensions and solutions. The Journal of Chemical Physics. 1952;20(4):571-.

22. Ajeel RK, Salim W-I, Hasnan K. Comparative study of the thermal performance of corrugated channels using ZnO-water nanofluid. Journal of Thermophysics and Heat Transfer. 2019;33(2):508-16.

23. HASAN MI, TBENA HL. Numerical investigation of microchannel heat sink with MEPCM suspension with different types of PCM. AL-Qadisiyah Journal for Engineering Sciences. 2018;11(1):115-33.

24. Manconi A, Tizzani P, Zeni G, Pepe S, Solaro G, editors. Simulated annealing and genetic algorithm optimization using comsol multiphysics: applications to the analysis of ground deformation in active volcanic areas. COMSOL Conference 2009; 2009.

25. Xoubi N, Soliman AY. Neutronic calculations of the IAEA 1OMW MTR benchmark reactor via finite element method using COMSOL multiphysics code. Annals of Nuclear Energy. 2017;109:277-83. doi:https://doi.org/10.1016/j.anucene.2017.05.033.

26. eK Germany O. Design for Reliability and Robustness through probabilistic Methods in COMSOL Multiphysics with OptiY. 
Figures

a)

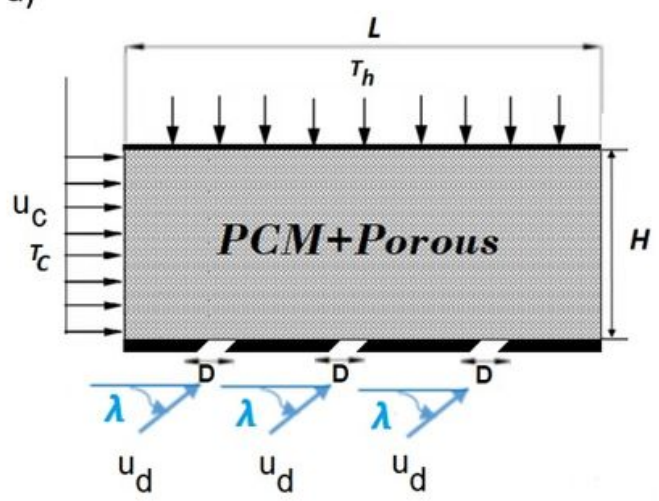

b)

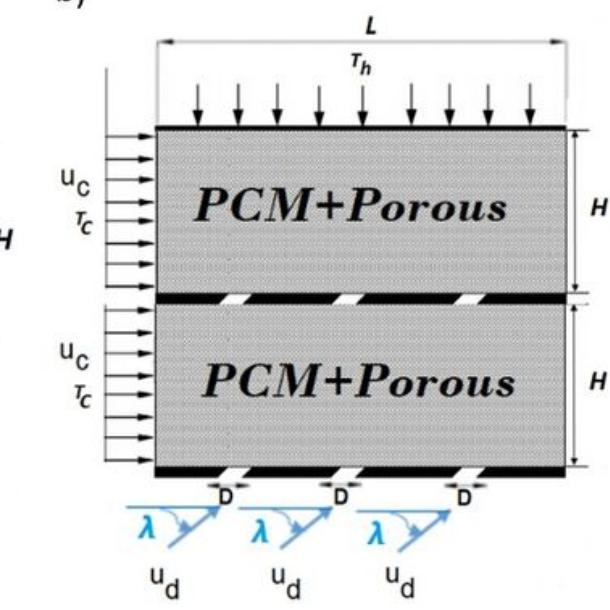

c)

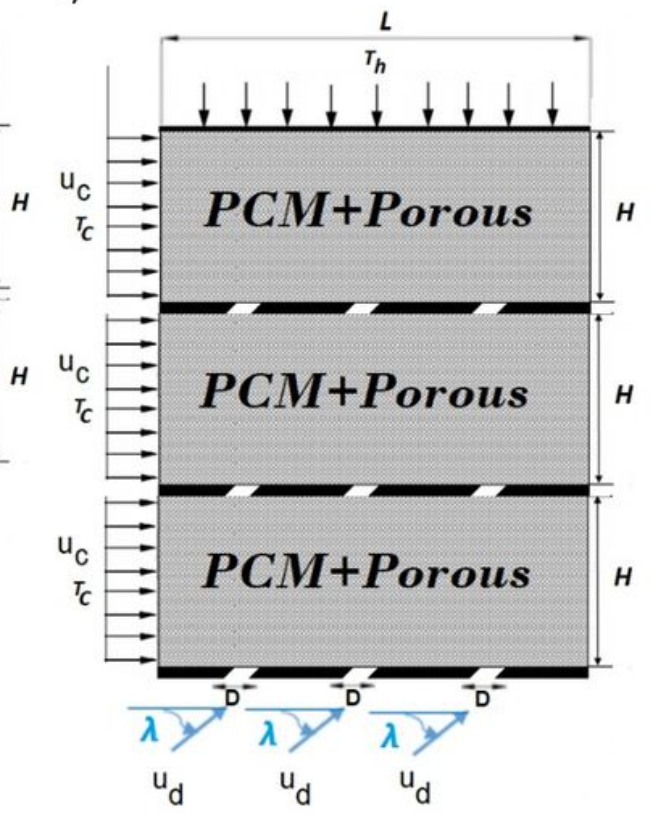

Figure 1

Schematic of microchannel a)one stage b) two stages c)three stages. 


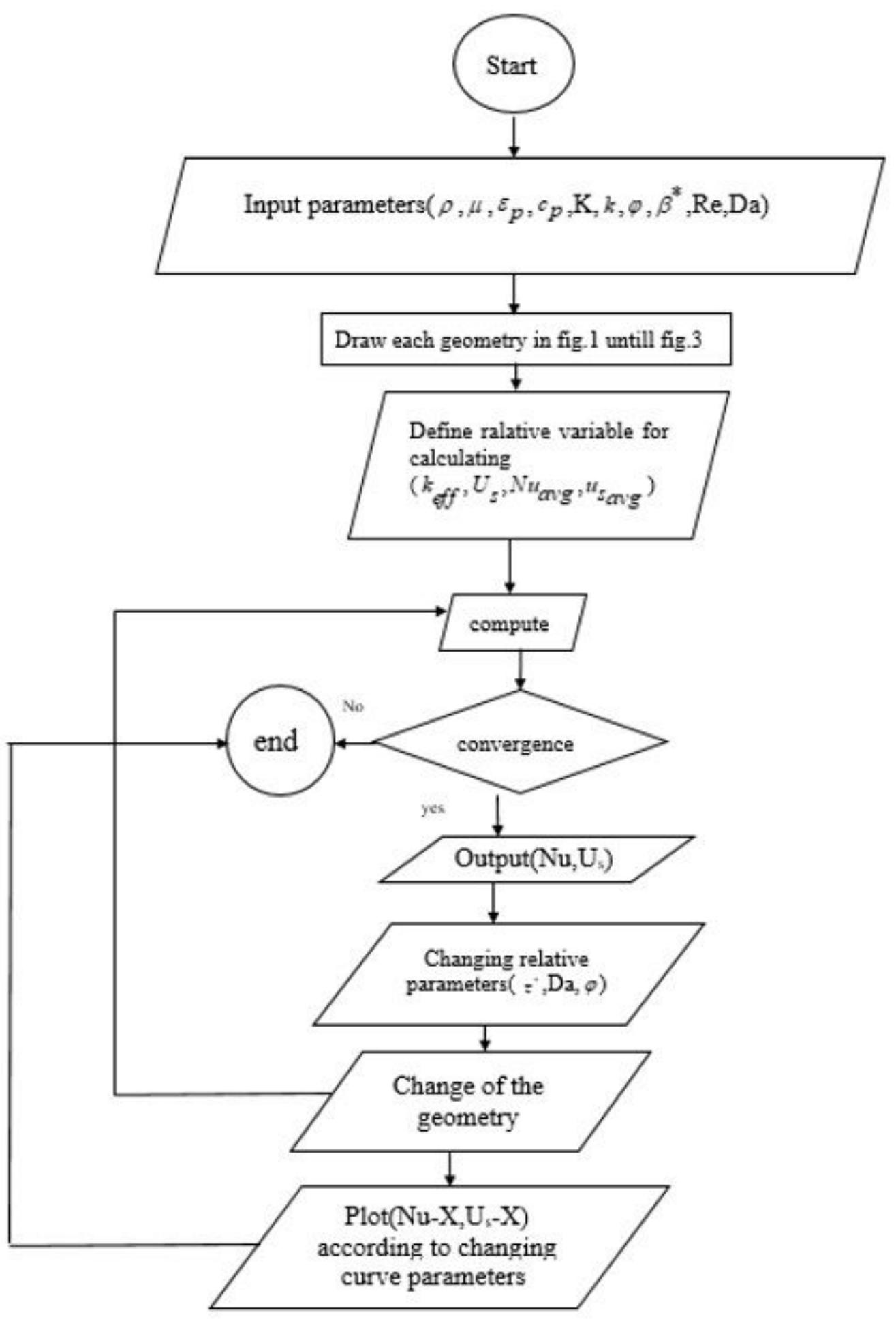

Figure 2

flowchart of methodology. 


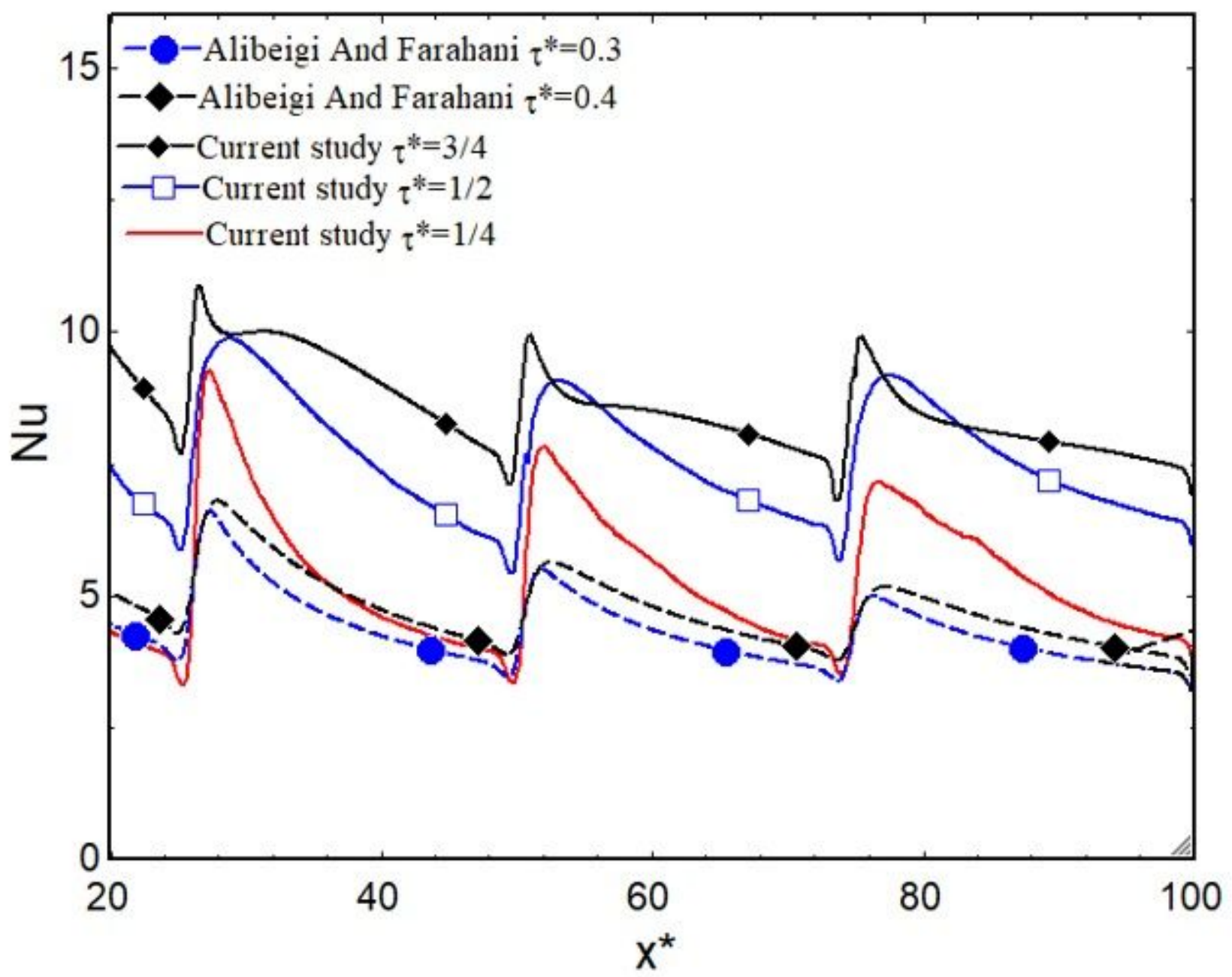

Figure 3

Validation of current study with similar study. 


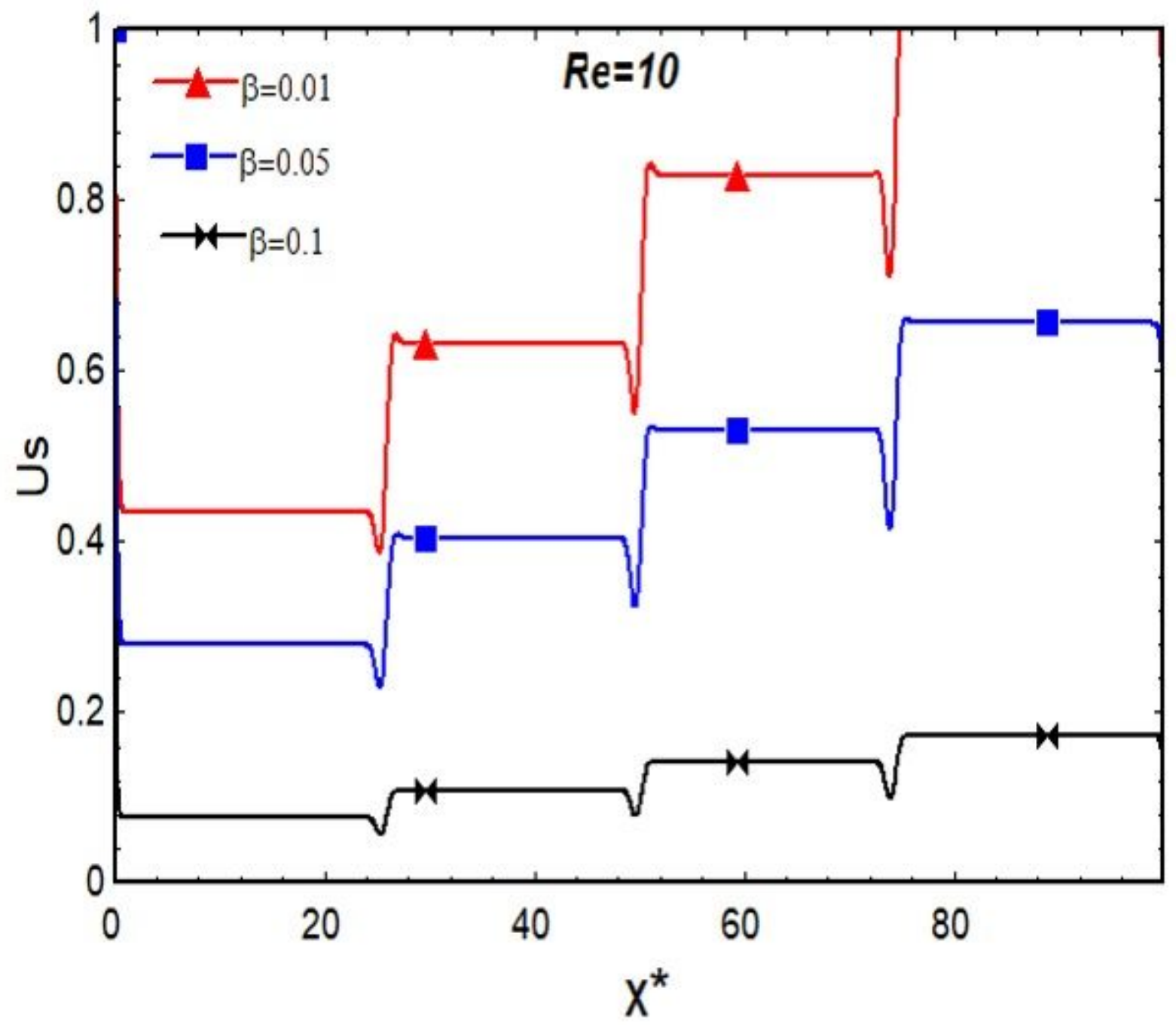

Figure 4

variations of slip velocity for $\mathrm{Re}=10$ in slip upper slip wall. 


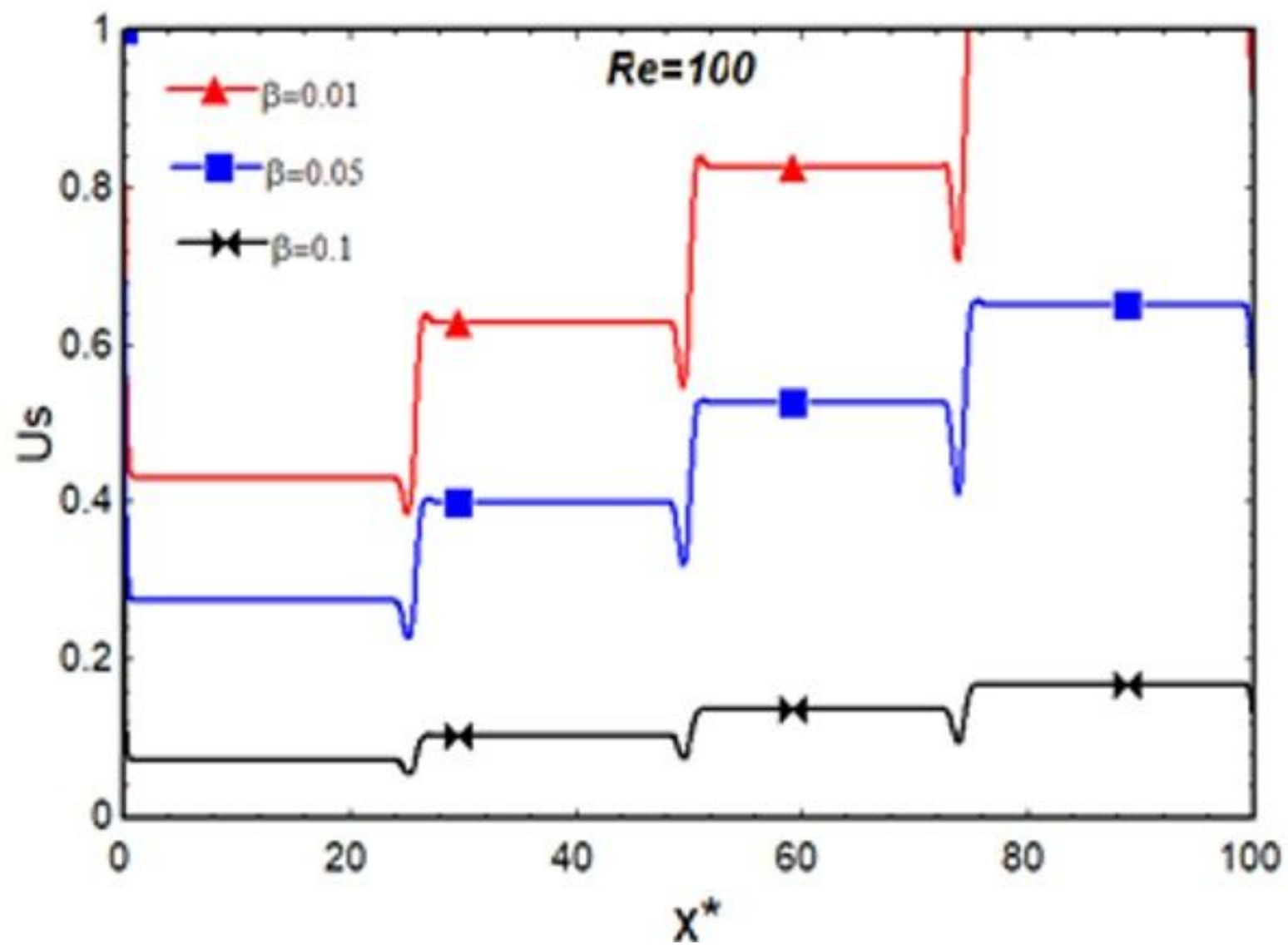

Figure 5

variations of slip velocity for $\mathrm{Re}=100$ in slip upper slip wall. 


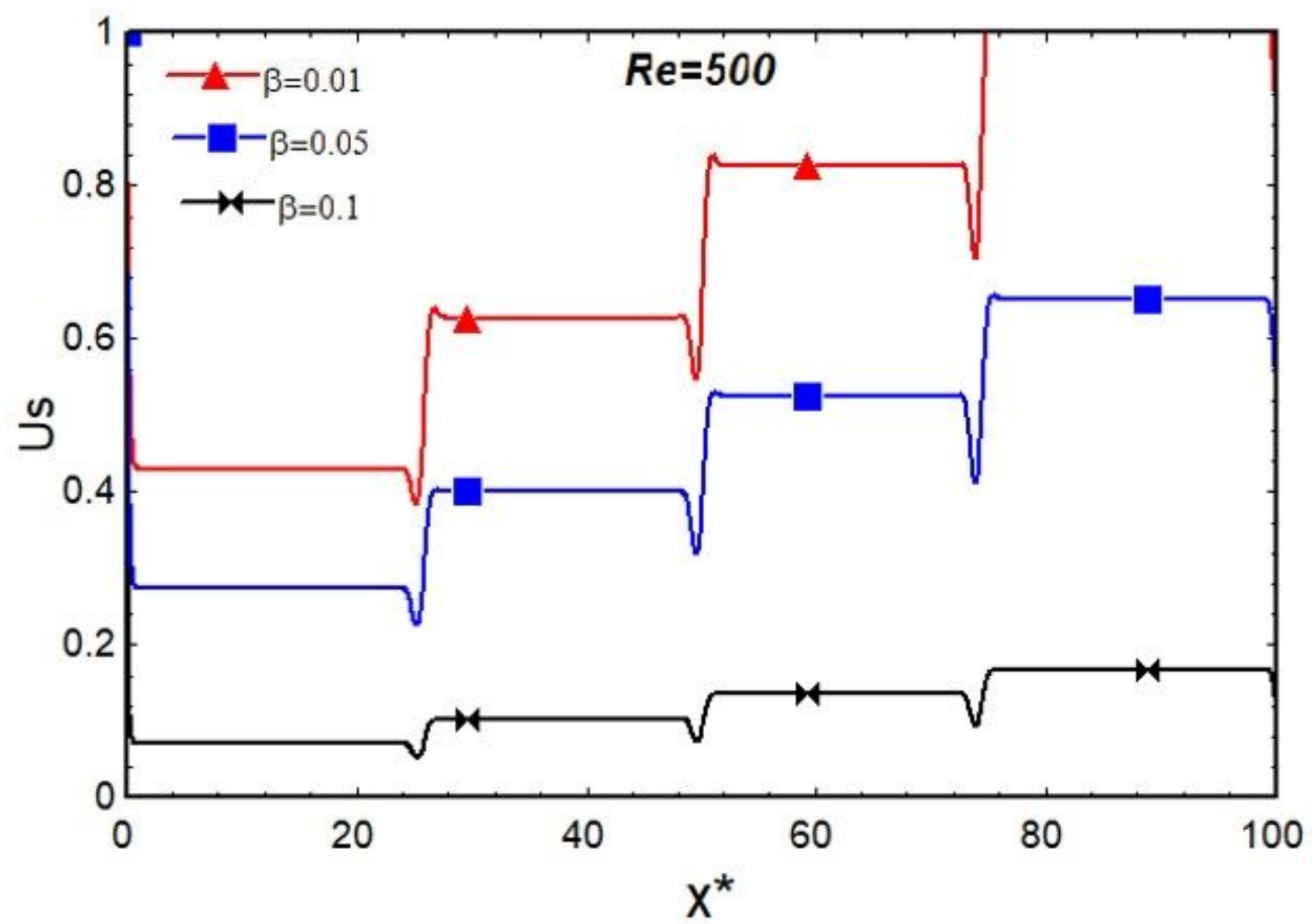

Figure 6

variations of slip velocity for $\mathrm{Re}=500$ in slip upper slip wall. 


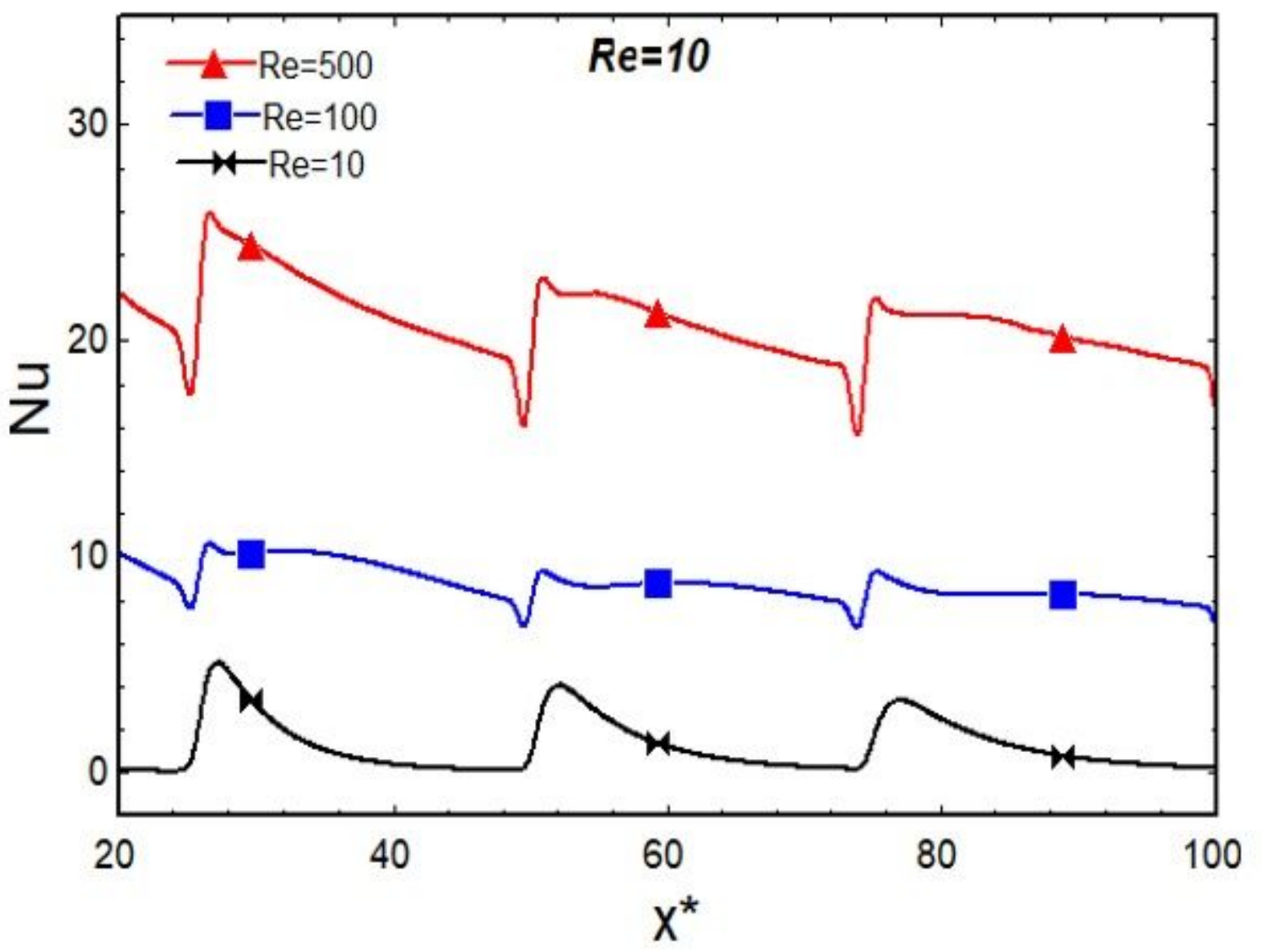

Figure 7

variations of Nusselt number for $\mathrm{Re}=10$ in constant temperature wall. 


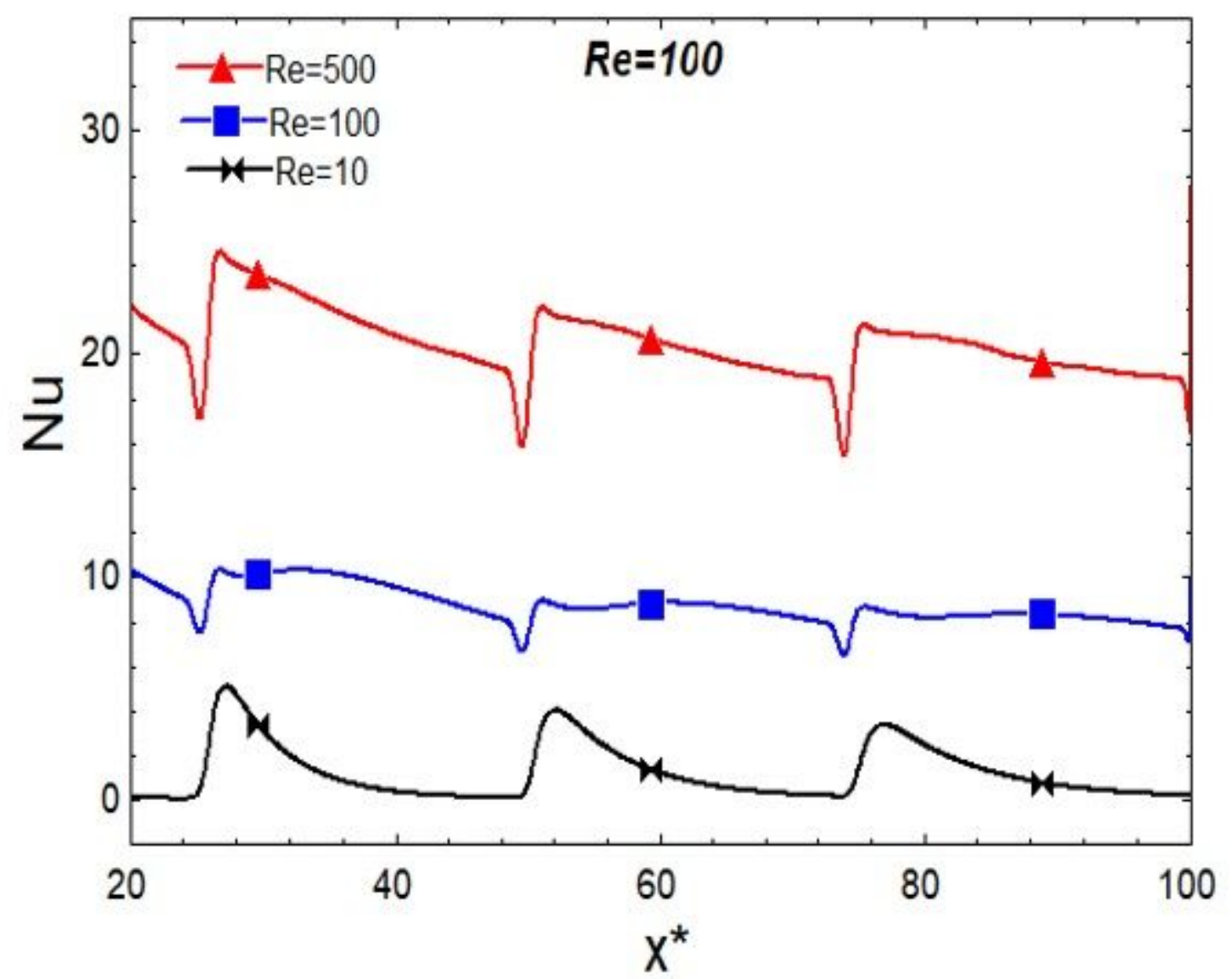

Figure 8

variations of Nusselt number for $\mathrm{Re}=100$ in constant temperature wall. 


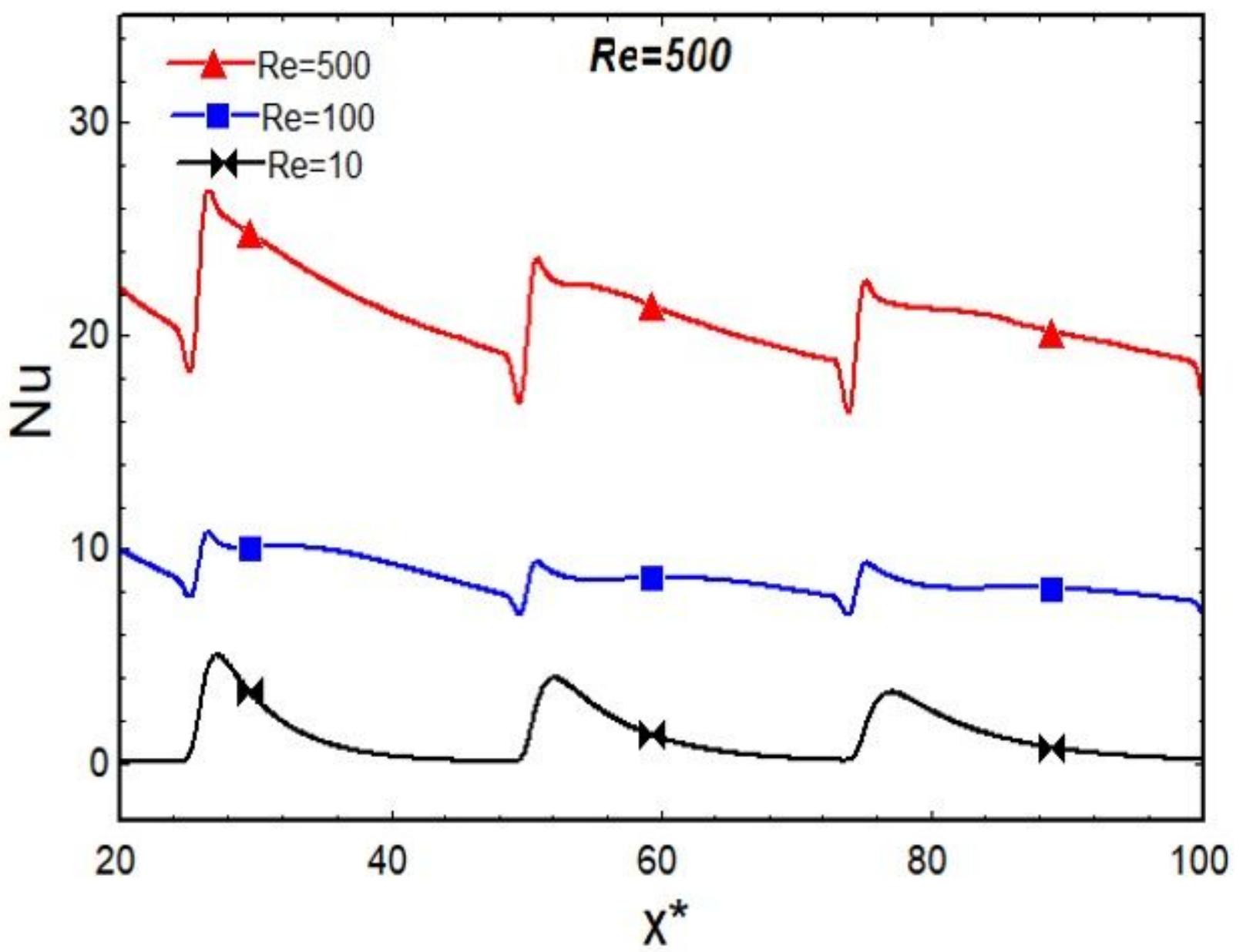

Figure 9

variations of Nusselt number for $\mathrm{Re}=500$ in constant temperature wall. 


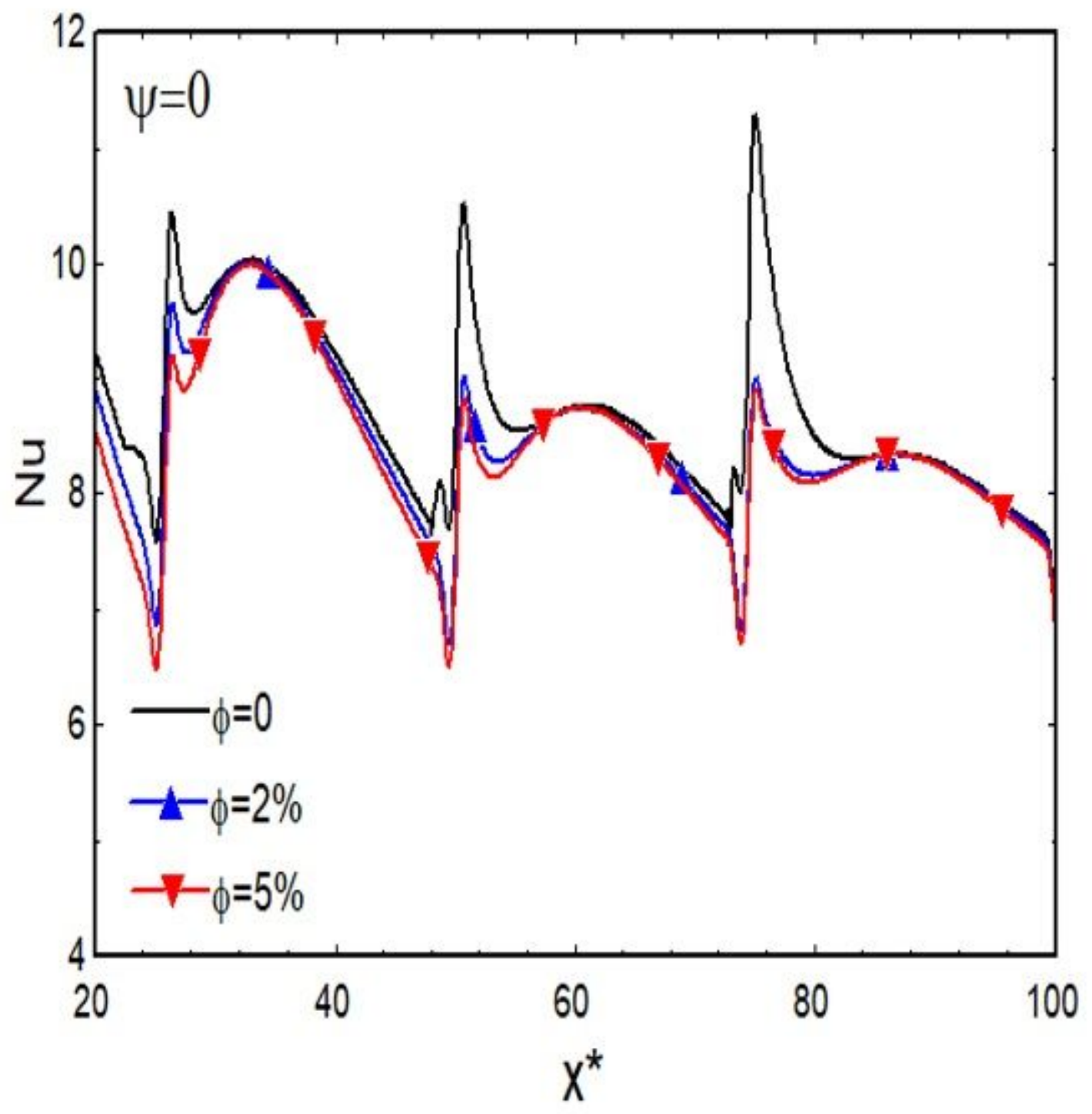

Figure 10

effects on nano particle volume fraction with 0 on Nusselt number. 


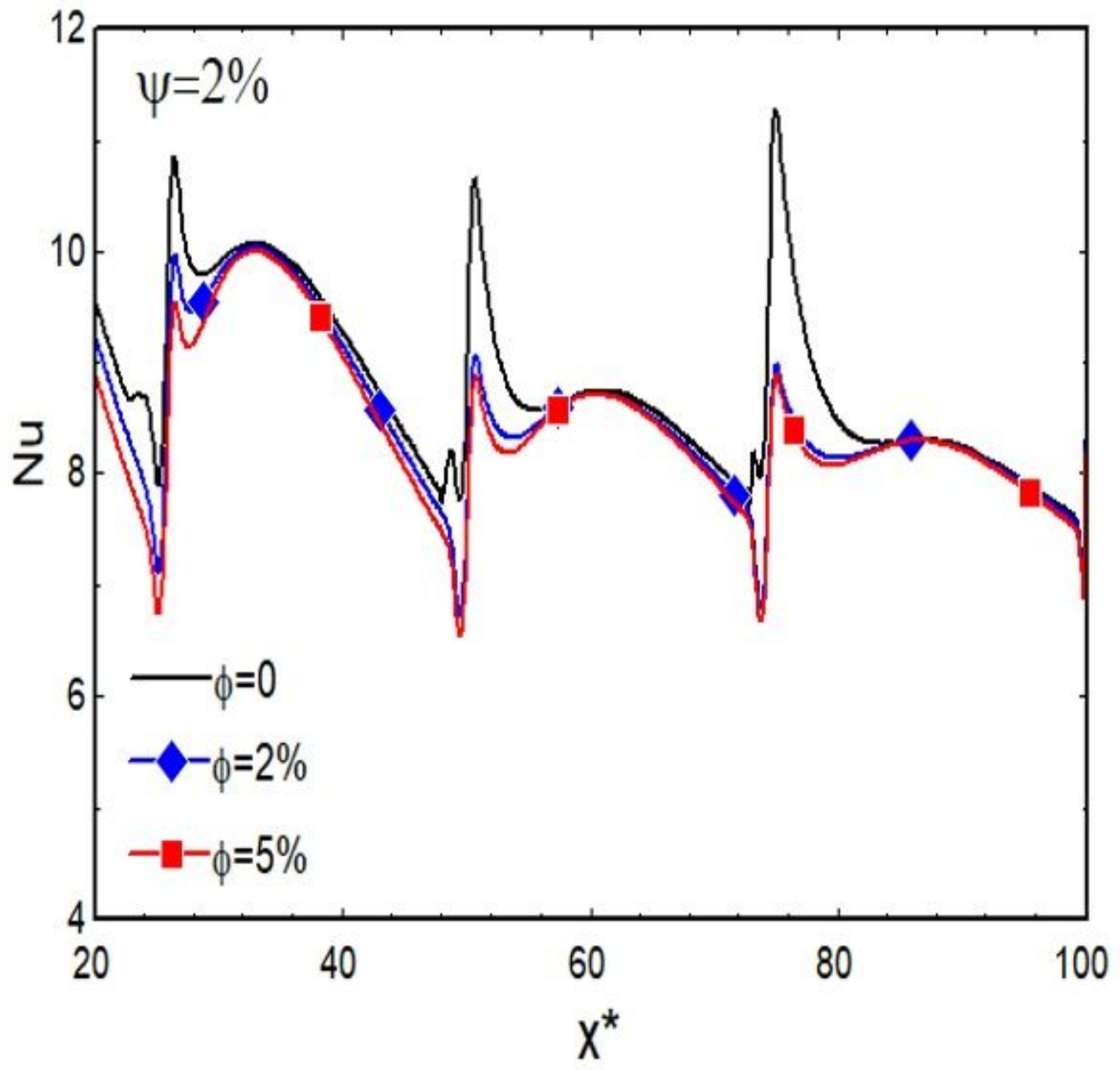

Figure 11

effects on nano particle volume fraction with $2 \%$ on Nusselt number. 


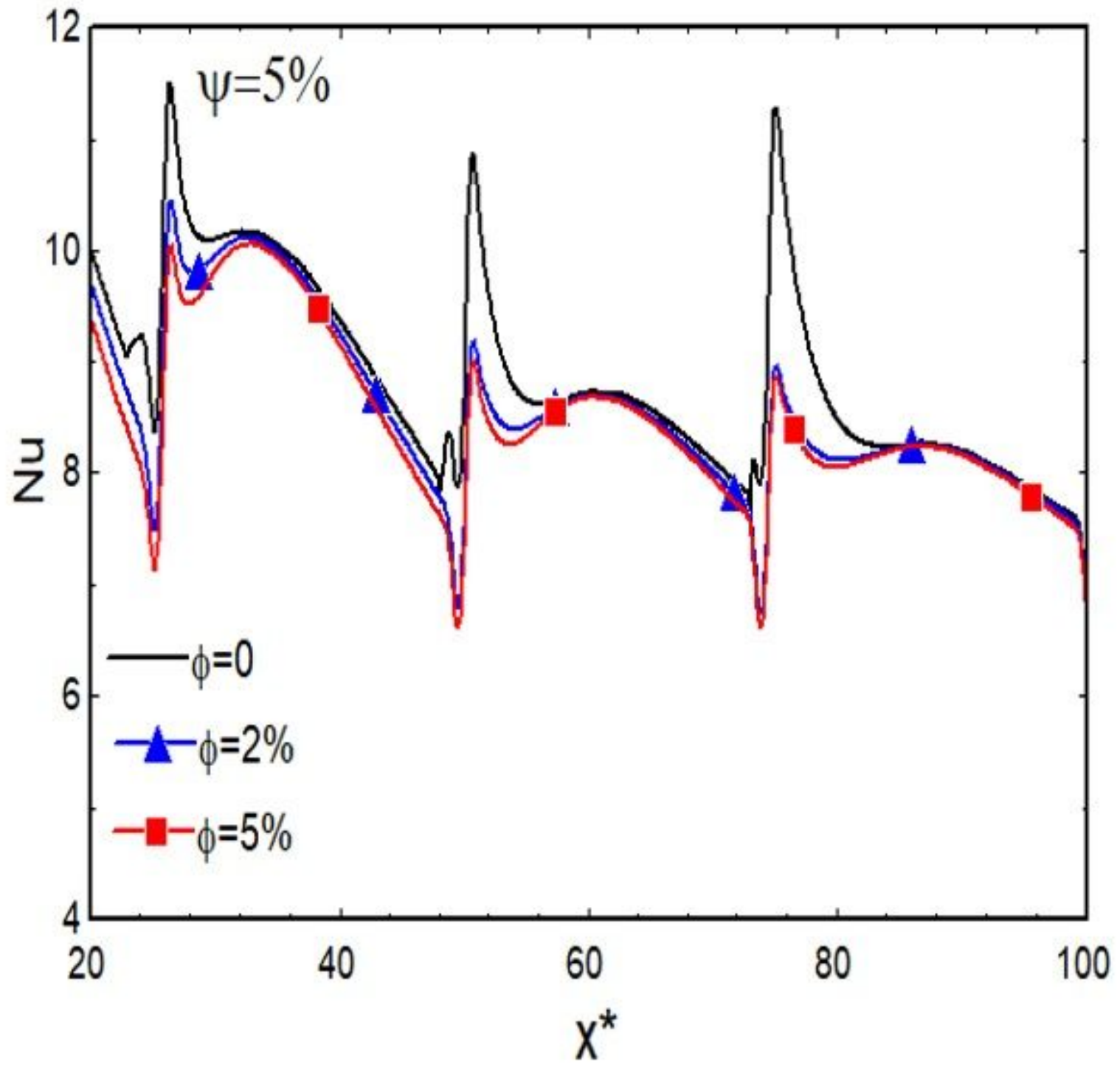

Figure 12

effects on nano particle volume fraction with $5 \%$ on Nusselt number. 


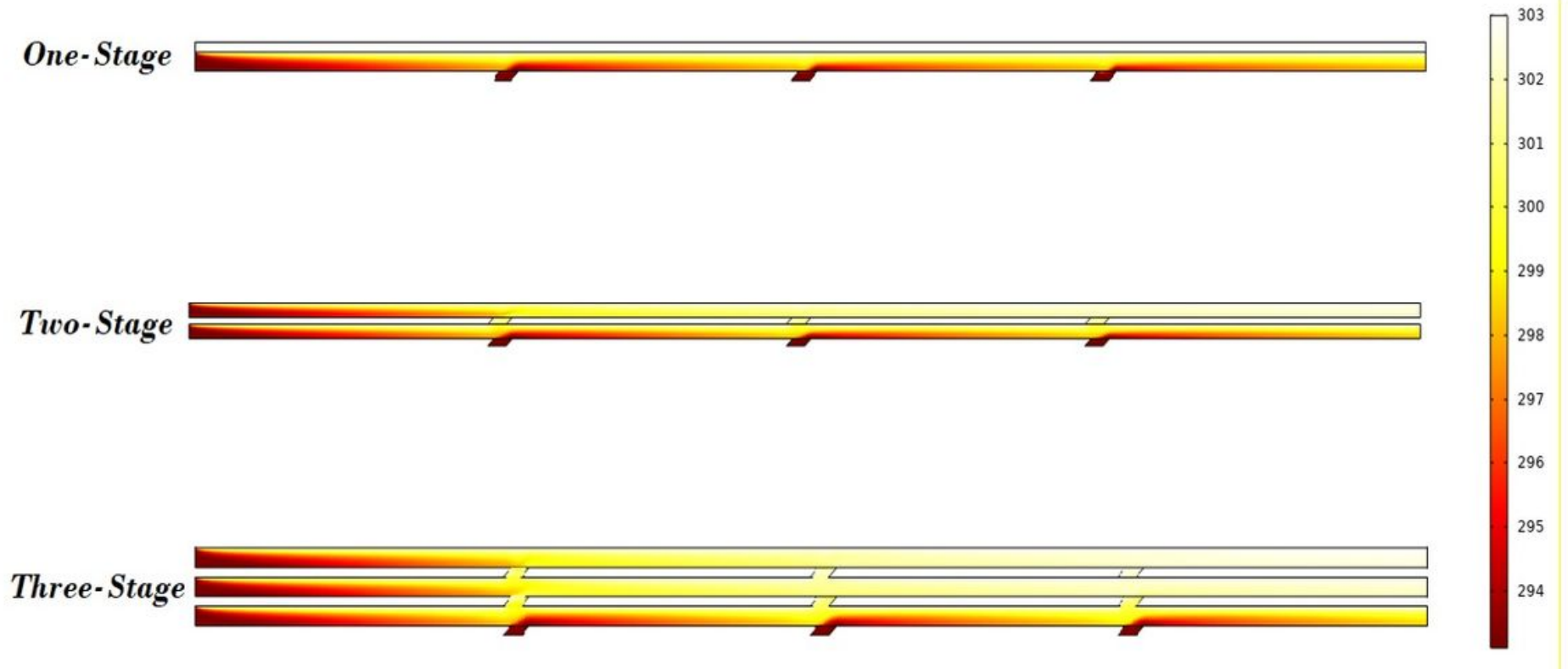

\section{Figure 13}

temperature counter for each stage. 